\title{
Marginal Performance of Corrected Ophthalmic Lenses
}

\author{
Francis E. Washer and Walter R. Darling
}

\begin{abstract}
The measured values of the departures of the marginal meridional and cylindrical powers from the corresponding measured values of the axial powers for 311 corrected ophthalmic lenses are reported. The spherical refractive powers of the lenses studied range from +7.00 to -20.00 diopters with cylindrical powers of 0.00 to 4.00 diopters. The probable errors of measurement are discussed. A set of tolerance values is suggested, and the degree of compliance with these suggested tolerances is shown.
\end{abstract}

\section{Introduction}

In recent years, the performance of spectacle lenses has been receiving growing attention. In 1951 , a study of the performance of spectacle lenses in the axial and extra-axial regions was initiated at the National Bureau of Standards at the request of the Veterans Administration. The objective of this investigation was the development of a set of tolerances to be used in purchase specifications. In order for these tolerances to be realistic, it was necessary to measure the refractive powers of a large number of spectacle lenses to determine what was currently feasible.

In a previous paper $[1],{ }^{1}$ the results of measurement of the axial performance of 311 spectacle lenses were reported. At present, several organizations are working in unison and attempting to produce a generally accepted standard for spectacle lenses, with emphasis being placed primarily on axial performance. It is probable that a similar effort will be initiated at some future time to extend the coverage of such a standard to include tolerances for marginal power. It therefore seems worthwhile to report the measured values of the departures of the extra-axial powers from the axial powers for the same 311 lenses. A tentative set of tolerances has also been prepared, and the degree of compliance with these tolerances is shown.

\section{Selection of Lenses for Measurement}

The lenses selected for measurement are the same ones whose axial performance was reported in a previous paper [1]. The present study is therefore confined to single-vision lenses of the type called "corrected ophthalmic lenses" [2]. Lenses of this kind have the total spherical and cylindrical powers divided between the front and rear surfaces in such a manner that the difference in axial and marginal power is minimized as far as practicable. Lenses of this variety are commonly known as members of a "corrected curve" series.

The powers of the lenses studied ranged from +7.00 to -20.00 diopters of spherical power and 0.00 to +6.00 diopters of cylindrical power. The major emphasis was placed on lenses having spherical

1 Figures in brackets indicate the literature references at the end of this 'paper. powers ranging from +7.00 to -6.00 diopters and cylindrical power ranging from 0.00 to +2.00 . The few reported that are outside this range are perhaps sufficient to indicate the probable performance in the region of higher powers. In order to make measurements on more than one lens of the same power, lenses of identical powers were purchased from five different manufacturers. Some power combinations were not readily available from each of the five, but generally at least three makers were able to supply lenses of like power. The final total of lenses that were measured was 311 , comprised of 3 to 5 lenses in each of 68 power combinations.

\section{Theory}

Spectacle lenses are normally specified in terms of spherical and cylindrical power as measured on the optical axis. The power of the lens, specified in this manner, is the best estimate of the correction required by the potential user. It is known, however, that the degree of correction afforded by a given lens varies as the eye rolls and looks successively through different areas of the lens. This cannot be avoided because the designer is constrained to work with but two surfaces and a single variety of glass at a time. If it were feasible to use two or more types of glass and a compound lens system in a spectacle lens, it might be possible to achieve a high degree of correction throughout the entire viewing area. This course of action is precluded by two principal factors, the first being excessive weight and the second being a substantial increase in the cost to the user. However, even for the simple lens type of construction, considerable improvement is effected by selection of the shape or form of the lens that yields best performance over the entire area.

To determine the difference in performance between the axial and marginal regions of a spectacle lens, it is customary to make all measurements with respect to the vertex sphere. The vertex sphere has its center at the center of rotation of the eye, and its surface is tangent to the rear surface of the spectacle lens at the point of intersection of the optical axis of the lens and its rear surface. The radius of the vertex sphere is usually accepted as being $27 \mathrm{~mm}$. When all measurements of axial and marginal powers are made with reference to the vertex sphere, comparisons can be readily made between the power at any specified extra-axial region and that at the optical axis. 

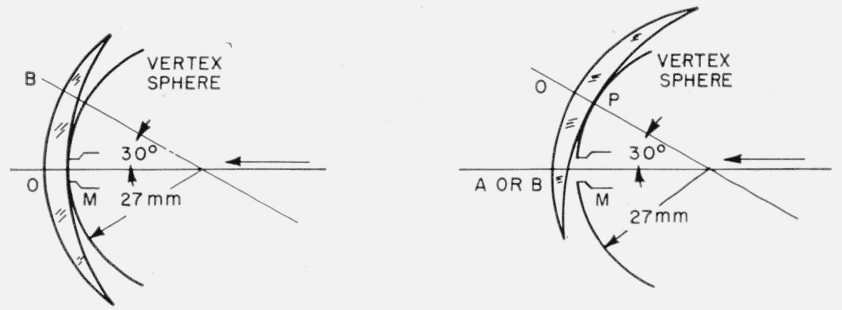

Figure 1. Schematic diagram showing the positions assumed by a spectacle lens when tested on a modified vertometer.

The left part of the figure shows arrangement for measurement of axial power. The fixed reference opening against which the lens is pressed is shown at $M$. The optical center $O$ is made to coincide with the center of $M$. The trace of the vertex sphere is also shown. In the right part of the figure, point $O$ is pressed against the vertex sphere as shown. Light from $M$ passes through the lens under test at points A or B and the marginal power can thus be measured with respect to the vertex sphere.

Figure 1 is a schematic diagram showing a spectacle lens in position for measurement with respect to the vertex sphere. The left-hand portion of the sketch shows the lens in position for axial measurements; the right-hand portion shows the lens in position for the measurement of marginal power in the region at $30^{\circ}$ from the axis. In attempting to compare the refractive powers in the marginal regions with that in the axial region, the use of the terms "spherical" and "cylindrical" power results in confusion. Consequently, in this paper, principal emphasis is placed on the "meridional power." The diagrammatic sketch, figure 2 , shows the regions where the powers

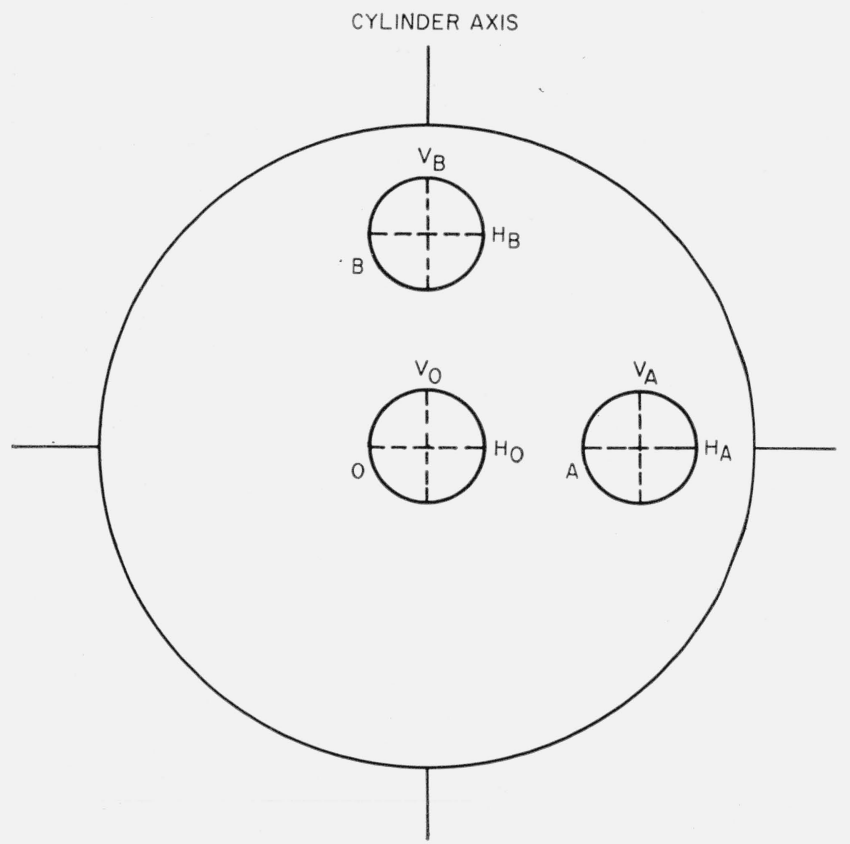

FIguRE 2. Schematic diagram showing the actual regions where measurements are made.

Each small area of the spectacle lens is treated as a separate lens and com parisons are made of the respective powers. The meridional axes of the various areas are as indicated. are measured. Measurements are made in the two principal meridians that yield the maximum and minimum meridional powers, respectively. In the figure, these meridians are designated by the symbols $V$ and $H$, with the subscripts $O, A$, and $B$, indicating the axial and marginal regions of the lens.

\subsection{Definitions}

For convenience, the definitions of all symbols used in describing the various powers and their interrelations are collected into this section. This system specifying the powers in various areas is patterned to some extent after the system devised by Whitwell and referred to by Emsley and Swaine [3].

$V_{P}=$ One of the principal meridional powers. It is given by the sum of the prescribed spherical and prescribed cylindrical power. It is the maximum meridional power when the prescribed powers are positive.

$H_{P}=$ The second principal meridional power. It is usually the same as the spherical power. It is the minimum meridional power when the prescribed powers are positive.

$C_{P}=$ Prescribed cylindrical power. It is the difference between the principal meridional powers and is given by the relation $C_{P}=V_{P}-H_{P}$.

$V_{o}=$ Measured value of one of the meridional powers at the optical center of the lens. It is the measured power for vertical lines.

$H_{O}=$ Measured value of the second meridional power at the optical center of the lens. It is the measured power for horizontal lines. (In the absence of cylindrical power, $V_{o}=H_{O}$.)

$C_{O}=$ The measured cylindrical power at the optical center of the lens. It is obtained from the relation, $\mathrm{C}_{O}=V_{O}-$ $H_{O}$.

$V_{A}, V_{B}=$ The measured marginal powers at points $A$ and $B$ in the same meridian as $V_{O}$ or parallel to the meridian of $V_{o}$. These are the measured marginal powers for vertical lines.

$H_{A}, H_{B}=$ The measured marginal powers at points $A$ and $B$ in the same meridian. as $H_{O}$ or parallel to the meridian of $H_{O}$. These are the measured marginal powers for horizontal lines.

$C_{A}=$ The measured cylindrical power at point $A$, given by the relation $C_{A}=V_{A}-H_{A}$.

$C_{B}=$ The measured cylindrical power at point $B$, given by the relation $C_{B}=V_{B}-H_{B}$.

\subsection{Additional Relations}

The following formulas are used in computing the departures of the measured values from the specified values for the axial region and the departures of the 
measured marginal values from the corresponding measured axial values:

$\begin{array}{lll}\Delta V_{o}=V_{o}-V_{P} & \Delta V_{A}=V_{A}-V_{o} & \Delta V_{B}=V_{B}-V_{o} \\ \Delta H_{o}=H_{o}-H_{P} & \Delta H_{A}=H_{A}-H_{O} & \Delta H_{B}=H_{B}-H_{O} \\ \Delta C_{o}=C_{O}-C_{P} & \Delta C_{A}=C_{A}-C_{O} & \Delta C_{B}=C_{B}-C_{O}\end{array}$

\section{Method of Measurement}

The axial and marginal powers of all lenses were measured on a standard vertex power-measuring instrument equipped with the modifying device described in a previous paper [2]. The axial powers were measured for the region shown at $O$ in figure 2 . It is evident from figure 2 that identical values of marginal power should be obtained for equivalent areas symmetrically located on either side of the axial point of the spectacle lens. Accordingly, in making the measurements of marginal power, it is customary to make and record five settings for both area $A$ and its equivalent area and a similar series for both area $B$ and its equivalent area. Some difference is found on opposite sides but it is usually sufficiently small that it can be neglected. The final accepted value for a given marginal power for a single observer represents an average for 10 settings. The measurements on each lens are made independently by each of three observers, and the final results are obtained by averaging the three sets. At the conclusion of the measurements, the values of the departures of the measured values from the specified values for the axial region and the departures of the measured marginal values from the corresponding measured axial values are determined with the aid of the equations given in section 3.2 .

\subsection{Variation in Measurements for Points $30^{\circ}$ From the Axis for Three Observers}

The determination of the refractive powers at $30^{\circ}$ from the axis is subject to considerably more uncertainty than those measured on the axis. The quality of definition is generally somewhat lower than on the axis, which tends to reduce the precision of the power determinations. In addition, any error in properly establishing the angle separating the area under test and the axis may produce appreciable error in some instances. For example, the change in meridional power with angle of viewing is likely to be small in the vicinity of the axis but may be changing rapidly with changing angle at $30^{\circ}$ from the axis. Consequently, a change of as little as \pm 0.5 degree in the region of $30^{\circ}$ may produce changes in the measured values of marginal power of as much as \pm 0.10 diopters. The depth of focus, throughout which the change in quality of definition of the image of the target reticle is not too apparent, is appreciably greater than that for axial depth of focus.

Because of these additional possibilities for error, an investigation of the reliability of the measurements at $30^{\circ}$ from the axis was also made for the three observers. Table 1 shows the values obtained
TABLE 1. Values of the deviations, $\Delta V_{A}, \Delta V_{B}, \Delta H_{A}, \Delta H_{B}$, $\Delta C_{A}$, and $\Delta C_{B}$, for six lenses obtained by each of three observers $(D, G$, and $B)$

The average values for the three observers are accepted as correct. All values are expressed in diopters.

\begin{tabular}{|c|c|c|c|c|c|c|c|c|}
\hline Obs. & Sph. & Cyl. & $\Delta V_{A}$ & $\Delta V_{B}$ & $\Delta H_{A}$ & $\Delta H_{B}$ & $\Delta C_{A}$ & $\Delta C_{B}$ \\
\hline $\begin{array}{l}D \\
G \\
G \\
B\end{array}$ & 7 & 2 & $\left\{\begin{array}{r}0.05 \\
.14 \\
.22\end{array}\right.$ & $\begin{array}{r}-0.02 \\
-.12 \\
-.14\end{array}$ & $\begin{array}{r}-0.29 \\
-.43 \\
-.41\end{array}$ & $\begin{array}{r}-0.37 \\
-.33 \\
-.29\end{array}$ & $\begin{array}{r}0.34 \\
.57 \\
.63\end{array}$ & $\begin{array}{r}0.35 \\
.21 \\
.15\end{array}$ \\
\hline Avg..... & $\ldots$ & $\ldots$ & 0.14 & -0.09 & -0.38 & -0.33 & 0.51 & 0.24 \\
\hline $\begin{array}{l}D \\
G \\
B \\
B\end{array}$ & 4 & 2 & $\left\{\begin{array}{r}-0.15 \\
-.13 \\
-.18\end{array}\right.$ & $\begin{array}{r}-0.31 \\
-.25 \\
-.40\end{array}$ & $\begin{array}{r}-0.30 \\
-.28 \\
-.34\end{array}$ & $\begin{array}{r}-0.40 \\
-.36 \\
-.51\end{array}$ & $\begin{array}{r}0.15 \\
.15 \\
.16\end{array}$ & $\begin{array}{r}0.09 \\
.11 \\
.11\end{array}$ \\
\hline Avg & $\ldots$ & $\ldots$ & -0.15 & -0.32 & -0.31 & -0.42 & 0.15 & 0.10 \\
\hline $\begin{array}{l}D \\
G \\
B \\
B\end{array}$ & 1 & 2 & $\left\{\begin{array}{r}+0.04 \\
.07 \\
.13\end{array}\right.$ & $\begin{array}{r}-0.13 \\
-.17 \\
-.13\end{array}$ & $\begin{array}{r}-0.09 \\
-.14 \\
-.06\end{array}$ & $\begin{array}{r}-0.26 \\
-.26 \\
-.20\end{array}$ & $\begin{array}{r}0.13 \\
.21 \\
.19\end{array}$ & $\begin{array}{r}0.13 \\
.09 \\
.07\end{array}$ \\
\hline Avg & $\ldots$ & -.- & 0.08 & -0.14 & -0.10 & -0.24 & 0.18 & 0.10 \\
\hline $\begin{array}{l}D \\
G \\
B \\
B\end{array}$ & 0 & 2 & $\left\{\begin{array}{r}0.08 \\
.17 \\
.16\end{array}\right.$ & $\begin{array}{l}-.09 \\
-.13 \\
-.08\end{array}$ & $\begin{array}{r}-0.02 \\
-.04 \\
-.03\end{array}$ & $\begin{array}{r}-0.14 \\
-.11 \\
-.15\end{array}$ & $\begin{array}{r}0.10 \\
.21 \\
.19\end{array}$ & $\begin{array}{r}0.05 \\
-.02 \\
+.07\end{array}$ \\
\hline Avg & $-\ldots$ & $\ldots$ & 0.14 & -0.10 & -0.03 & -0.13 & 0.17 & 0.03 \\
\hline $\begin{array}{l}D \\
G \\
B \\
B\end{array}$ & -1 & 2 & $\left\{\begin{array}{l}0.16 \\
.17 \\
.21\end{array}\right.$ & $\begin{array}{r}-0.16 \\
-.17 \\
-.14\end{array}$ & $\begin{array}{r}-0.05 \\
-.04 \\
-.03\end{array}$ & $\begin{array}{r}-0.21 \\
-.13 \\
-.10\end{array}$ & $\begin{array}{r}0.21 \\
.21 \\
.24\end{array}$ & $\begin{array}{r}0.05 \\
-.04 \\
-.04\end{array}$ \\
\hline Avg .- & -... & -..- & 0.18 & -0.16 & -0.04 & -0.15 & 0.22 & -0.01 \\
\hline $\begin{array}{l}D \\
G \\
B \\
B\end{array}$ & -4 & 2 & $\left\{\begin{array}{r}0.37 \\
.30 \\
.30\end{array}\right.$ & $\begin{array}{r}+0.17 \\
.04 \\
.13\end{array}$ & $\begin{array}{r}+0.26 \\
.22 \\
.20\end{array}$ & $\begin{array}{r}+0.10 \\
.06 \\
.02\end{array}$ & $\begin{array}{r}0.11 \\
.08 \\
.10\end{array}$ & $\begin{array}{r}+0.07 \\
-.02 \\
+.11\end{array}$ \\
\hline Avg..... & -... & -... & 0.32 & 0.11 & 0.23 & 0.06 & 0.10 & 0.05 \\
\hline $\begin{array}{l}D \\
G \\
B \\
B\end{array}$ & -6 & 2 & $\left\{\begin{array}{l}0.49 \\
.41 \\
.48\end{array}\right.$ & $\begin{array}{r}0.27 \\
.32 \\
.29\end{array}$ & $\begin{array}{r}0.41 \\
.41 \\
.49\end{array}$ & $\begin{array}{r}0.12 \\
.20 \\
.15\end{array}$ & $\begin{array}{r}0.08 \\
.00 \\
-.01\end{array}$ & $\begin{array}{r}0.15 \\
.12 \\
.14\end{array}$ \\
\hline Avg $\ldots$ & -... & $\ldots$ & 0.46 & 0.29 & 0.44 & 0.16 & 0.02 & 0.14 \\
\hline
\end{tabular}

by each of the three observers, identified as $D, G$, and $B$, for a representative group of six lenses. The fourth line in each case is the average value obtained and is the one accepted as correct. The symbols, $\Delta V_{A}, \Delta V_{B}, \Delta H_{A}, \Delta H_{B}, \Delta C_{A}$, and $\Delta C_{B}$, signify respectively the departure of the various powers at points $A$ and $B$ at $30^{\circ}$ from the axis from the corresponding measured quantities on the axis. It may be noted that the departures from the average for each observer, although small, are appreciably greater than for measurements on the axis [1].

The results of measurements, made at $30^{\circ}$ off-axis on 36 lenses of varied powers by 3 observers, were analysed to determine the magnitude of observer bias or systematic error. The results of this analysis are shown in table 2 . The departures from the accepted average measured-power deviation were determined for each of the 3 observers for each of the 36 lenses.

The average departure was computed for each observer and is taken as a measure of the systematic error or bias for each observer. It is apparent that

TABLE 2. Systematic differences in the values of $\Delta V_{A}, \Delta V_{B}$, $\Delta H_{A}, \Delta H_{B}, \Delta C_{A}$, and $\Delta C_{B}$ for three observers, $D, G$, and $B$, based on measurements at $30^{\circ}$ off-axis for 36 lenses The differences are given in diopters.

\begin{tabular}{|c|c|c|c|c|c|c|}
\hline \multirow{2}{*}{ Observer } & \multicolumn{6}{|c|}{ A verage departure from the average of- } \\
\hline & $\Delta V_{A}$ & $\Delta V_{B}$ & $\Delta H_{A}$ & $\Delta H_{B}$ & $\Delta C_{A}$ & $\Delta C_{B}$ \\
\hline $\begin{array}{l}D \\
G \\
B\end{array}$ & $\begin{array}{r}-0.013 \\
-.003 \\
+.016\end{array}$ & $\begin{array}{r}+0.006 \\
-.012 \\
+.009\end{array}$ & $\begin{array}{r}+0.007 \\
-.005 \\
+.001\end{array}$ & $\begin{array}{r}-0.008 \\
+.006\end{array}$ & $\begin{array}{r}-0.016 \\
-.003 \\
+.019\end{array}$ & $\begin{array}{r}+0.014 \\
-.024 \\
+.001\end{array}$ \\
\hline$B \ldots$ & +.016 & +.009 & +.001 & & +.019 & +.001 \\
\hline
\end{tabular}


so far as the determinations of $\Delta V$ and $\Delta H$ are concerned, the magnitudes of the systematic error are of the same order of magnitude as found for $V$ and $H$ on the axis [1]. The systematic errors in $\Delta C$ are greater at the margins than on the axis but are nevertheless small. It can therefore be assumed that the contribution of observer bias to measurements of $\Delta V_{A}, \Delta V_{B}, \Delta H_{A}$, and $\Delta H_{B}$, will not usually exceed \pm 0.01 diopter. In addition, the contribution from the same source to measurements of $\Delta C_{A}$ and $\Delta C_{B}$ will not usually exceed \pm 0.02 diopter. In view of the small magnitudes of observer bias and the probable error of a single determination for each observer, it can reasonably be claimed that the error of determinations of $\Delta V_{A}, \Delta V_{B}, \Delta H_{A}, \Delta H_{B}, \Delta C_{A}$, and $\Delta C_{B}$ will generally be less than \pm 0.03 diopter.

Table 3 shows a frequency distribution of the errors for the 3 observers in the determination of the marginal power of the 36 lenses. For example, in the determination of $\Delta V_{A}$, the results of measurement by observer $D$ are within \pm 0.03 diopter of the accepted average value of $\Delta V_{A}$ for 53 percent of the lenses measured. It is also clear that for any of the observers, the likelihood of being in error by more than \pm 0.06 diopter is quite small.

TABLE 3. Frequency distribution of departures from the average value of $\Delta V_{A}, \Delta V_{B}, \Delta H_{A}, \Delta H_{B}, \Delta C_{A}$, and $\Delta C_{B}$ for each of three observers

The results are based on measurements of 36 lenses. All values are in diopters

A. Percentage of determinations that do not depart from average value of $\Delta V_{A}$ (or $\left.\Delta V_{B}\right)$ by more than $\delta V_{A}\left(\right.$ or $\left.\delta V_{B}\right)$ for observer-

\begin{tabular}{|c|c|c|c|c|c|c|c|}
\hline \multirow{2}{*}{$\delta V_{A}$} & \multicolumn{3}{|c|}{$\Delta V_{A}$} & \multirow{2}{*}{$\delta V_{B}$} & \multicolumn{3}{|c|}{$\Delta V_{B}$} \\
\hline & $D$ & $G$ & $B$ & & $D$ & $G$ & $B$ \\
\hline $\begin{array}{r} \pm 0.03 \\
.06 \\
.09 \\
.12 \\
.15\end{array}$ & $\begin{array}{r}53 \\
92 \\
100 \\
----\end{array}$ & $\begin{array}{r}84 \\
97 \\
100 \\
---\end{array}$ & $\begin{array}{r}61 \\
89 \\
100 \\
--. \\
-.\end{array}$ & $\begin{array}{r} \pm 0.03 \\
.06 \\
.09 \\
.12 \\
.15\end{array}$ & $\begin{array}{r}58 \\
75 \\
97 \\
97 \\
100\end{array}$ & $\begin{array}{r}53 \\
84 \\
94 \\
97 \\
100\end{array}$ & $\begin{array}{r}67 \\
81 \\
89 \\
92 \\
100\end{array}$ \\
\hline
\end{tabular}

B. Percentage of determinations that do not depart from average value of $\Delta H_{A}$ (or $\Delta H_{B}$ ) by more than $\delta H_{A}$ (or $\delta H_{B}$ ) for observer-

\begin{tabular}{|c|c|c|c|c|c|c|c|}
\hline \multirow{2}{*}{$\Delta H_{A}$} & \multicolumn{3}{|c|}{$\Delta H_{A}$} & \multirow{2}{*}{$\delta H_{B}$} & \multicolumn{3}{|c|}{$\Delta H_{B}$} \\
\hline & $D$ & $G$ & $B$ & & $D$ & G & $B$ \\
\hline $\begin{array}{r} \pm 0.03 \\
.06 \\
.09 \\
.12 \\
.15\end{array}$ & $\begin{array}{r}69 \\
92 \\
100 \\
--- \\
--\end{array}$ & $\begin{array}{r}75 \\
91 \\
100 \\
--. \\
--\end{array}$ & $\begin{array}{r}75 \\
94 \\
97 \\
100 \\
---\end{array}$ & $\begin{array}{r} \pm 0.03 \\
.06 \\
.09 \\
.12 \\
.15\end{array}$ & $\begin{array}{r}56 \\
86 \\
94 \\
94 \\
100\end{array}$ & $\begin{array}{r}72 \\
97 \\
97 \\
100 \\
-\end{array}$ & $\begin{array}{r}47 \\
81 \\
94 \\
100 \\
-\ldots\end{array}$ \\
\hline
\end{tabular}

C. Percentage of determinations that do not depart from average value of $\Delta C_{A}$ (or $\left.\Delta C_{B}\right)$ by more than $\delta C_{A}\left(\right.$ or $\left.\delta C_{B}\right)$ for observer-

\begin{tabular}{|c|c|c|c|c|c|c|c|}
\hline \multirow{2}{*}{$\delta C_{A}$} & \multicolumn{3}{|c|}{$\Delta C_{A}$} & \multirow{2}{*}{$\delta C_{B}$} & \multicolumn{3}{|c|}{$\Delta C_{B}^{\prime}$} \\
\hline & $D$ & $G$ & $B$ & & $D$ & $G$ & $B$ \\
\hline $\begin{array}{r} \pm 0.03 \\
.06 \\
.09 \\
.12 \\
.18\end{array}$ & $\begin{array}{r}42 \\
83 \\
97 \\
97 \\
100\end{array}$ & $\begin{array}{r}69 \\
97 \\
100 \\
--- \\
---\end{array}$ & $\begin{array}{r}44 \\
72 \\
94 \\
97 \\
100\end{array}$ & $\begin{array}{r} \pm 0.03 \\
.06 \\
.09 \\
.12 \\
.18\end{array}$ & $\begin{array}{r}39 \\
75 \\
86 \\
97 \\
100\end{array}$ & $\begin{array}{r}47 \\
72 \\
91 \\
100 \\
--\end{array}$ & $\begin{array}{r}49 \\
75 \\
86 \\
92 \\
100\end{array}$ \\
\hline
\end{tabular}

\section{Results of Measurement on 311 Spectacle Lenses}

The values of $V_{A}, H_{A}, V_{B}, H_{B}, V_{O}$, and $H_{O}$ were measured for the entire group of 311 lenses. From these measured values the values of $C_{O}, C_{A}$, and $C_{B}$ were determined. For the present purposes, the values of the departures of the measured values on axis from the prescribed axial values and the values of the measured marginal values from the measured. axial values are of greater interest than the actual values of marginal and axial meridional power. The values of $\Delta V_{o}, \Delta H_{o}$, and $\Delta C_{o}$ are reported in an earlier paper [1]. In the present paper, the empha sis is given to the measured values of $\Delta V_{A}, \Delta H_{A}, \Delta C_{A}$, $\Delta V_{B}, \Delta H_{B}$, and $\Delta C_{B}$. The results of these determina tions are given in tables 4 to 10 . The order of the lenses in these tables is the same as that given in the earlier paper to facilitate comparison. It is eivdent from a cursory examination, that the values of $\Delta V_{A}, \Delta H_{A}, \Delta C_{A}, \Delta V_{B}, \Delta H_{B}$, and $\Delta C_{B}$ are frequently quite large, particularly for the higher spherical and cylindrical powers.

\section{Establishment of Tolerances}

The establishment of tolerances for marginal power for spectacle lenses is somewhat more complex than is the case for axial power. The interrelation of the various powers in three areas instead of the single central area has to be kept constantly in mind. The performance in the peripheral regions of spectacle lenses made in accordance with recognized best practice is generally not equal to that commonly obtained in the axial regions. So for tolerances to serve a useful purpose, their magtitude must be made appreciably greater than those acceptable on axis, but at the same time, they should be held as small as feasible to insure optimum performance under the circumstances.

\subsection{Proposed Tolerances for Marginal Powers}

A careful analysis of the magnitudes of the deviations, $\Delta V_{A}, \Delta V_{B}, \Delta H_{A}, \Delta H_{B}, \Delta C_{A}$, and $\Delta C_{B}$, that are listed in tables 4 to 10 , was made. On the basis of this analysis, tolerances were established for use in a purchase specification [4]. These tolerances are specified as follows:

(a) The marginal meridional powers referred to the vertex sphere at points $A$ and $B$, located as shown in figure 2 , shall not depart from the measured axial meridional powers by amounts in excess of those values of the tolerances for meridional power shown in tables 11 to 15 .

(b) The cylindrical power, referred to the vertex sphere at points $A$ and $B$ shall not depart from the measured axial cylindrical powers by an amount in excess of those values of the tolerances shown in tables 11 to 15 . 
TABLE 4. Measured values of the deviations in meridional power at the marginal points $A$ and $B$ from the measured axial power for 72 spectacle lenses having zero cylindrical power and with spherical power varying from +7.00 to -18.00 diopters

The nominal prescribed spherical powers are listed under "Sph". The significance of the symbols at the heads of the columns is given in section 3.2 and figure 2. All values are expressed in diopters.

\begin{tabular}{|c|c|c|c|c|c|c|}
\hline Sph. & $\Delta V_{A}$ & $\Delta H_{A}$ & $\Delta C_{A}$ & $\Delta V_{B}$ & $\Delta H_{B}$ & $\Delta C_{B}$ \\
\hline 7.00 & $\left\{\begin{array}{r}-0.17 \\
-.25 \\
-.22\end{array}\right.$ & $\begin{array}{r}0.23 \\
-.05 \\
+.02\end{array}$ & $\begin{array}{r}-0.40 \\
-.20 \\
-.24\end{array}$ & $\begin{array}{r}0.23 \\
-.04 \\
+.02\end{array}$ & $\begin{array}{r}-0.18 \\
-.24 \\
-.21\end{array}$ & $\begin{array}{r}0.41 \\
.20 \\
.23\end{array}$ \\
\hline f. 00 & $\left\{\begin{array}{r}-0.04 \\
-.20 \\
-.01 \\
-.09 \\
-.06\end{array}\right.$ & $\begin{array}{r}-0.17 \\
-.24 \\
-.19 \\
-.18 \\
-.25\end{array}$ & $\begin{array}{r}+0.13 \\
.04 \\
.18 \\
.09 \\
.19\end{array}$ & $\begin{array}{r}-0.19 \\
-.22 \\
-.22 \\
-.20 \\
-.26\end{array}$ & $\begin{array}{r}-0.04 \\
-.20 \\
-.02 \\
-.10 \\
-.06\end{array}$ & $\begin{array}{r}-0.15 \\
-.02 \\
-.20 \\
-.10 \\
-.20\end{array}$ \\
\hline 5.00 & $\left\{\begin{array}{r}-0.24 \\
-.09 \\
-.18 \\
+.02 \\
-.10\end{array}\right.$ & $\begin{array}{r}-0.26 \\
-.15 \\
-.22 \\
-.16 \\
-.19\end{array}$ & $\begin{array}{r}0.02 \\
.06 \\
.04 \\
.18 \\
.09\end{array}$ & $\begin{array}{r}-0.21 \\
-.19 \\
-.18 \\
-.18 \\
-.22\end{array}$ & $\begin{array}{r}-0.23 \\
-.15 \\
-.15 \\
+.03 \\
-.10\end{array}$ & $\begin{array}{r}+0.02 \\
-.04 \\
-.03 \\
-.21 \\
-.12\end{array}$ \\
\hline 4. 00 & $\left\{\begin{array}{r}-0.12 \\
+.03 \\
.01 \\
-.020 \\
-.13\end{array}\right.$ & $\begin{array}{r}-0.14 \\
-.08 \\
-.07 \\
-.07 \\
-.13\end{array}$ & $\begin{array}{r}0.02 \\
.11 \\
.08 \\
.07 \\
.00\end{array}$ & $\begin{array}{r}-0.14 \\
-.08 \\
-.02 \\
-.31 \\
-.17\end{array}$ & $\begin{array}{r}-0.13 \\
+.03 \\
.02 \\
-.19 \\
-.17\end{array}$ & $\begin{array}{r}-0.01 \\
-.11 \\
-.04 \\
-.12 \\
.00\end{array}$ \\
\hline 3.00 & $\left\{\begin{array}{r}-0.10 \\
+.02 \\
.00 \\
-.24 \\
-.18\end{array}\right.$ & $\begin{array}{r}-0.13 \\
-.08 \\
-.07 \\
-.00 \\
-.15\end{array}$ & $\begin{array}{r}0.03 \\
.10 \\
.07 \\
-.04 \\
-.03\end{array}$ & $\begin{array}{r}-0.10 \\
-.07 \\
=.08 \\
-.20 \\
-.12\end{array}$ & $\begin{array}{r}-0.13 \\
+.01 \\
-.00 \\
-.21 \\
-.14\end{array}$ & $\begin{array}{r}+0.03 \\
-.08 \\
-.08 \\
+.01 \\
.02\end{array}$ \\
\hline 2.00 & $\left\{\begin{array}{r}-0.12 \\
-.03 \\
-.07 \\
-.05 \\
-.04\end{array}\right.$ & $\begin{array}{r}-0.09 \\
-.08 \\
-.08 \\
-.07 \\
-.03\end{array}$ & $\begin{array}{r}-0.03 \\
+.05 \\
.01 \\
.02 \\
-.01\end{array}$ & $\begin{array}{r}-0.10 \\
-.08 \\
-.07 \\
-.08 \\
-.03\end{array}$ & $\begin{array}{r}-0.08 \\
-.05 \\
-.08 \\
-.05 \\
-.02\end{array}$ & $\begin{array}{r}-0.02 \\
-.03 \\
+.01 \\
-.03 \\
-.01\end{array}$ \\
\hline 1.00 & $\left\{\begin{array}{r}-0.02 \\
-.02 \\
-.03 \\
-.01 \\
-.04\end{array}\right.$ & $\begin{array}{r}-0.01 \\
-.01 \\
-.02 \\
-.12 \\
-.05\end{array}$ & $\begin{array}{r}-0.01 \\
-.01 \\
-.01 \\
+.11 \\
+.01\end{array}$ & $\begin{array}{r}-0.01 \\
-.00 \\
-.03 \\
-.02 \\
-.03\end{array}$ & $\begin{array}{r}-0.02 \\
-.01 \\
-.02 \\
-.01 \\
-.05\end{array}$ & $\begin{array}{r}+0.01 \\
.01 \\
-.01 \\
-.01 \\
+.02\end{array}$ \\
\hline 0.00 & $\left\{\begin{array}{r}+0.03 \\
.00 \\
-.01 \\
-.02 \\
+.01\end{array}\right.$ & $\begin{array}{r}0.00 \\
.00 \\
.00 \\
+.02 \\
-.01\end{array}$ & $\begin{array}{r}0.03 \\
.00 \\
-.01 \\
-.04 \\
.02\end{array}$ & $\begin{array}{r}-0.05 \\
+.01 \\
.00 \\
.02 \\
-.01\end{array}$ & $\begin{array}{r}+0.02 \\
.01 \\
-.01 \\
-.01 \\
+.01\end{array}$ & $\begin{array}{r}-0.07 \\
+.00 \\
.01 \\
.03 \\
-.02\end{array}$ \\
\hline-1.00 & $\left\{\begin{array}{r}0.00 \\
-.03 \\
-.01 \\
.00 \\
+.02\end{array}\right.$ & $\begin{array}{r}+0.06 \\
.05 \\
.08 \\
.04 \\
.05\end{array}$ & $\begin{array}{r}-0.06 \\
-.08 \\
-.09 \\
-.04 \\
-.03\end{array}$ & $\begin{array}{r}+0.08 \\
.01 \\
.06 \\
.05 \\
.06\end{array}$ & $\begin{array}{r}0.02 \\
-.06 \\
-.01 \\
+.00 \\
.01\end{array}$ & $\begin{array}{r}+0.06 \\
.07 \\
.07 \\
.05 \\
.05\end{array}$ \\
\hline-2.00 & $\left\{\begin{array}{r}0.02 \\
-.09 \\
+.09 \\
.11 \\
.06\end{array}\right.$ & $\begin{array}{r}0.09 \\
.02 \\
.14 \\
.13 \\
.19\end{array}$ & $\begin{array}{r}-0.07 \\
-.11 \\
-.05 \\
-.02 \\
-.13\end{array}$ & $\begin{array}{r}0.08 \\
.02 \\
.11 \\
.16 \\
.15\end{array}$ & $\begin{array}{r}0.04 \\
-.08 \\
+.11 \\
.12 \\
.06\end{array}$ & $\begin{array}{r}0.04 \\
.10 \\
.00 \\
.04 \\
.09\end{array}$ \\
\hline-3.00 & $\left\{\begin{array}{r}0.09 \\
.06 \\
.11 \\
.14 \\
.05\end{array}\right.$ & $\begin{array}{l}0.15 \\
.08 \\
.17 \\
.16 \\
.15\end{array}$ & $\begin{array}{r}-0.06 \\
-.02 \\
-.06 \\
-.02 \\
-.10\end{array}$ & $\begin{array}{r}0.14 \\
.10 \\
.17 \\
.18 \\
.18\end{array}$ & $\begin{array}{r}0.08 \\
.03 \\
.16 \\
.13 \\
.02\end{array}$ & $\begin{array}{r}0.06 \\
.07 \\
.01 \\
.05 \\
.16\end{array}$ \\
\hline-4.00 & $\left\{\begin{array}{r}0.14 \\
.15 \\
.03 \\
-.07 \\
+.17\end{array}\right.$ & $\begin{array}{r}0.19 \\
.26 \\
.17 \\
.16 \\
.20\end{array}$ & $\begin{array}{r}-0.05 \\
-.11 \\
-.14 \\
-.23 \\
-.03\end{array}$ & $\begin{array}{l}0.20 \\
.25 \\
.19 \\
.15 \\
.20\end{array}$ & $\begin{array}{r}0.13 \\
.15 \\
.01 \\
-.06 \\
+.17\end{array}$ & $\begin{array}{r}0.07 \\
.10 \\
.18 \\
.21 \\
.03\end{array}$ \\
\hline-6.00 & $\begin{array}{r}0.31 \\
.45 \\
.35 \\
.25 \\
.22\end{array}$ & $\begin{array}{r}0.32 \\
.33 \\
.32 \\
.22 \\
.02\end{array}$ & $\begin{array}{r}-0.01 \\
+.12 \\
\cdot \quad 03 \\
.03 \\
.20\end{array}$ & $\begin{array}{r}0.30 \\
.32 \\
.24 \\
.18 \\
.03\end{array}$ & $\begin{array}{r}0.30 \\
.39 \\
.30 \\
.24 \\
.20\end{array}$ & $\begin{array}{r}0.00 \\
-.07 \\
-.06 \\
-.06 \\
-.17\end{array}$ \\
\hline-10.00 & $\begin{array}{r}0.34 \\
.16 \\
.21\end{array}$ & $\begin{array}{r}0.30 \\
.01 \\
.00\end{array}$ & $\begin{array}{r}0.04 \\
.15 \\
.21\end{array}$ & $\begin{array}{r}0.36 \\
.02 \\
.00\end{array}$ & $\begin{array}{r}0.42 \\
.15 \\
.20\end{array}$ & $\begin{array}{r}-0.06 \\
-.13 \\
-.20\end{array}$ \\
\hline-14.00 & $\left\{\begin{array}{r}0.27 \\
-.04 \\
+.45\end{array}\right.$ & $\begin{array}{r}0.26 \\
.25 \\
.57\end{array}$ & $\begin{array}{r}0.01 \\
-.29 \\
-.12\end{array}$ & $\begin{array}{r}0.24 \\
.24 \\
.59\end{array}$ & $\begin{array}{r}0.24 \\
-.02 \\
+.46\end{array}$ & $\begin{array}{r}0.00 \\
+.26 \\
.13\end{array}$ \\
\hline-18.00 & $\left\{\begin{array}{r}0.26 \\
-.24 \\
+.18\end{array}\right.$ & $\begin{array}{r}0.22 \\
-.02 \\
+.30\end{array}$ & $\begin{array}{r}+0.04 \\
-.22 \\
-.12\end{array}$ & $\begin{array}{r}.022 \\
-.02 \\
+.30\end{array}$ & $\begin{array}{r}0.17 \\
-.23 \\
+.22\end{array}$ & $\begin{array}{r}0.04 \\
.21 \\
.08\end{array}$ \\
\hline
\end{tabular}

TABLE 5. Measured values of the deviations in meridional power at the marginal points $A$ and $B$ from the measured axial power for 76 spectacle lenses having a cylindrical nower of +1.00 diopter and with spherical power varying from +6.00 to -19.00 diopters

The nominal prescribed spherical powers are listed under "Sph". The signifi. cance of the symbols at the heads of the columns is given in section 3.2 and figure 2. All values are expressed in diopters.

\begin{tabular}{|c|c|c|c|c|c|c|}
\hline Sph. & $\triangle V_{A}$ & $\triangle H_{A}$ & $\triangle C_{A}$ & $\triangle V_{B}$ & $\triangle H_{B}$ & $\triangle C_{B}$ \\
\hline 7.00 & $\begin{array}{r}0.06 \\
.40 \\
-.13\end{array}$ & $\begin{array}{r}-0.28 \\
-.35 \\
-.35\end{array}$ & $\begin{array}{r}0.34 \\
.75 \\
.22\end{array}$ & $\begin{array}{r}-0.20 \\
-.18 \\
+.07\end{array}$ & $\begin{array}{r}0.08 \\
.21 \\
-.34\end{array}$ & $\begin{array}{r}-0.28 \\
-.39 \\
+.41\end{array}$ \\
\hline 6.00 & $\begin{array}{r}0.00 \\
+.22 \\
.02 \\
-.02 \\
-.01\end{array}$ & $\begin{array}{r}-0.35 \\
-.27 \\
-.33 \\
-.36 \\
-.33\end{array}$ & $\begin{array}{r}0.35 \\
.49 \\
.35 \\
.34 \\
.32\end{array}$ & $\begin{array}{r}-0.20 \\
-.08 \\
-.14 \\
-.20 \\
-.19\end{array}$ & $\begin{array}{r}-0.11 \\
+.15 \\
-.19 \\
-.11 \\
-.06\end{array}$ & $\begin{array}{r}-0.09 \\
-.23 \\
+.05 \\
-.09 \\
-.13\end{array}$ \\
\hline 5.00 & $\left\{\begin{array}{r}-0.09 \\
-.06 \\
-.11 \\
-.10 \\
+.05\end{array}\right.$ & $\begin{array}{r}-0.37 \\
-.28 \\
-.34 \\
-.30 \\
-.28\end{array}$ & $\begin{array}{r}0.28 \\
.22 \\
23 \\
.20 \\
.33\end{array}$ & $\begin{array}{r}-0.12 \\
-.15 \\
-.18 \\
-.21 \\
-.13\end{array}$ & $\begin{array}{r}-0.15 \\
-.18 \\
-.19 \\
-.10 \\
.00\end{array}$ & $\begin{array}{r}+0.03 \\
.03 \\
.01 \\
-.11 \\
-.13\end{array}$ \\
\hline 4. 00 & $\left\{\begin{array}{r}-0.13 \\
-.17 \\
-.05 \\
.00 \\
-.07\end{array}\right.$ & $\begin{array}{l}-.15 \\
-.22 \\
-.01 \\
+.07 \\
-.16\end{array}$ & $\begin{array}{r}0.02 \\
.05 \\
-.04 \\
-.07 \\
+.09\end{array}$ & $\begin{array}{r}-0.19 \\
-.05 \\
-.01 \\
-.02 \\
-.01\end{array}$ & $\begin{array}{r}-0.27 \\
-.09 \\
-.21 \\
-.15 \\
-.21\end{array}$ & $\begin{array}{r}+0.08 \\
.04 \\
.20 \\
.13 \\
.20\end{array}$ \\
\hline 3.00 & $\left\{\begin{array}{r}-0.23 \\
-.17 \\
+.06 \\
.03 \\
.00\end{array}\right.$ & $\begin{array}{r}-0.28 \\
-.25 \\
-.17 \\
-.13 \\
-.16\end{array}$ & $\begin{array}{r}0.05 \\
.08 \\
.23 \\
.16 \\
.16\end{array}$ & $\begin{array}{r}-0.15 \\
-.07 \\
-.04 \\
+.01 \\
-.04\end{array}$ & $\begin{array}{r}-0.20 \\
-.13 \\
+.01 \\
.00 \\
.01\end{array}$ & $\begin{array}{r}0.05 \\
.06 \\
-.05 \\
+.01 \\
-.05\end{array}$ \\
\hline 2.00 & $\begin{array}{r}0.04 \\
-.01 \\
-.01 \\
-.13 \\
-.02\end{array}$ & $\begin{array}{r}+0.01 \\
-.08 \\
-.13 \\
-.13 \\
.00\end{array}$ & $\begin{array}{r}0.03 \\
.07 \\
.12 \\
.00 \\
-.02\end{array}$ & $\begin{array}{r}0.03 \\
-.09 \\
-.16 \\
-.01 \\
-.08\end{array}$ & $\begin{array}{r}-0.03 \\
-.18 \\
-.15 \\
-.08 \\
-.16\end{array}$ & $\begin{array}{r}+0.06 \\
.09 \\
-.01 \\
+.07 \\
.08\end{array}$ \\
\hline 1.00 & $\left\{\begin{array}{r}-0.15 \\
+.04 \\
-.17 \\
+.02 \\
.01\end{array}\right.$ & $\begin{array}{r}-0.13 \\
-.15 \\
-.08 \\
-.03 \\
-.10\end{array}$ & $\begin{array}{r}-0.02 \\
+.19 \\
-.09 \\
+.05 \\
.11\end{array}$ & $\begin{array}{r}+0.01 \\
.04 \\
.05 \\
-.02 \\
-.09\end{array}$ & $\begin{array}{r}-0.11 \\
-.04 \\
-.03 \\
-.10 \\
-.11\end{array}$ & $\begin{array}{r}0.12 \\
.08 \\
.08 \\
.08 \\
.02\end{array}$ \\
\hline 0.00 & $\left\{\begin{array}{r}0.00 \\
.05 \\
-.08 \\
-.07 \\
-.09\end{array}\right.$ & $\begin{array}{r}-0.07 \\
-.05 \\
-.07 \\
-.10 \\
-.05\end{array}$ & $\begin{array}{r}0.07 \\
.10 \\
-.01 \\
+.03 \\
-.04\end{array}$ & $\begin{array}{r}-0.08 \\
-.04 \\
+.04 \\
.07 \\
.08\end{array}$ & $\begin{array}{r}-0.09 \\
-.04 \\
-.03 \\
-.05 \\
-.01\end{array}$ & $\begin{array}{r}0.01 \\
.00 \\
.07 \\
.12 \\
.09\end{array}$ \\
\hline-1.00 & $\left\{\begin{array}{r}-0.04 \\
-.01 \\
+.08 \\
.09 \\
-.03\end{array}\right.$ & $\begin{array}{r}-0.02 \\
-.01 \\
-.02 \\
+.02 \\
-.03\end{array}$ & $\begin{array}{r}-0.02 \\
.00 \\
+.10 \\
.07 \\
.00\end{array}$ & $\begin{array}{r}0.08 \\
.11 \\
-.07 \\
-.05 \\
+.11\end{array}$ & $\begin{array}{r}-0.05 \\
-.01 \\
-.03 \\
-.02 \\
-.02\end{array}$ & $\begin{array}{r}0.13 \\
.12 \\
-.04 \\
-.03 \\
+.13\end{array}$ \\
\hline-2.00 & $\left\{\begin{array}{r}+0.16 \\
-.08 \\
-.04 \\
+.03 \\
.15\end{array}\right.$ & $\begin{array}{r}+0.07 \\
.00 \\
-.02 \\
+.01 \\
.04\end{array}$ & $\begin{array}{r}0.09 \\
-.08 \\
-.02 \\
+.02 \\
.11\end{array}$ & $\begin{array}{r}0.02 \\
.17 \\
.11 \\
.19 \\
.01\end{array}$ & $\begin{array}{r}0.06 \\
-.02 \\
-.04 \\
+.04 \\
.00\end{array}$ & $\begin{array}{r}-0.04 \\
+.19 \\
.15 \\
.15 \\
.01\end{array}$ \\
\hline-3.00 & $\left\{\begin{array}{r}0.11 \\
.10 \\
.06 \\
.15 \\
.05\end{array}\right.$ & $\begin{array}{r}0.02 \\
.08 \\
.00 \\
.14 \\
.09\end{array}$ & $\begin{array}{r}0.09 \\
.02 \\
.06 \\
.01 \\
-.04\end{array}$ & $\begin{array}{r}0.19 \\
.23 \\
.22 \\
.25 \\
.24\end{array}$ & $\begin{array}{r}0.05 \\
.10 \\
.06 \\
.19 \\
.12\end{array}$ & $\begin{array}{r}0.14 \\
.13 \\
.16 \\
.06 \\
.12\end{array}$ \\
\hline-4.00 & $\begin{array}{r}0.30 \\
.25 \\
.24 \\
.07 \\
.23\end{array}$ & $\begin{array}{r}0.19 \\
.22 \\
.08 \\
.09 \\
-.04\end{array}$ & $\begin{array}{r}+0.11 \\
.03 \\
.16 \\
-.02 \\
+.27\end{array}$ & $\begin{array}{r}0.21 \\
.16 \\
.09 \\
.18 \\
.00\end{array}$ & $\begin{array}{r}0.15 \\
.14 \\
.13 \\
.06 \\
.09\end{array}$ & $\begin{array}{r}0.06 \\
.02 \\
-.04 \\
+.12 \\
-.09\end{array}$ \\
\hline-6.00 & $\begin{array}{r}0.23 \\
.34 \\
.41 \\
.33 \\
-.05\end{array}$ & $\begin{array}{r}-0.04 \\
+.25 \\
.41 \\
.33 \\
.16\end{array}$ & $\begin{array}{r}0.27 \\
.09 \\
.00 \\
.00 \\
-.21\end{array}$ & $\begin{array}{r}0.06 \\
.34 \\
.39 \\
.29 \\
.23\end{array}$ & $\begin{array}{r}0.12 \\
.22 \\
.23 \\
.26 \\
.16\end{array}$ & $\begin{array}{r}-0.06 \\
+.12 \\
.16 \\
.03 \\
.07\end{array}$ \\
\hline-10.00 & $\left\{\begin{array}{r}+0.79 \\
.72 \\
.22 \\
-.20\end{array}\right.$ & $\begin{array}{l}0.37 \\
.41 \\
.38 \\
.05\end{array}$ & $\begin{array}{r}+0.42 \\
.31 \\
-.16 \\
-.25\end{array}$ & $\begin{array}{r}0.57 \\
.57 \\
.43 \\
.20\end{array}$ & $\begin{array}{r}0.58 \\
.56 \\
.40 \\
-.02\end{array}$ & $\begin{array}{r}-0.01 \\
+.01 \\
.03 \\
.22\end{array}$ \\
\hline-11.00 & $\left\{\begin{array}{r}+0.20 \\
.22 \\
.33\end{array}\right.$ & $\begin{array}{r}0.17 \\
.67 \\
.29\end{array}$ & $\begin{array}{r}0.03 \\
-.45 \\
+.04\end{array}$ & $\begin{array}{r}0.16 \\
.31 \\
.10\end{array}$ & $\begin{array}{r}+0.10 \\
.39 \\
.31\end{array}$ & $\begin{array}{r}0.06 \\
-.08 \\
-.21\end{array}$ \\
\hline-15.00 & $\left\{\begin{array}{r}-0.18 \\
+.66 \\
.32\end{array}\right.$ & $\begin{array}{r}0.11 \\
.61 \\
.43\end{array}$ & $\begin{array}{r}-.29 \\
+.05 \\
-.11\end{array}$ & $\begin{array}{r}0.09 \\
.47 \\
.25\end{array}$ & $\begin{array}{r}-0.41 \\
+.70 \\
.24\end{array}$ & $\begin{array}{r}0.50 \\
-.23 \\
+.01\end{array}$ \\
\hline-19.00 & $\left\{\begin{array}{r}-0.22 \\
+.48 \\
.17\end{array}\right.$ & $\begin{array}{r}0.10 \\
.40 \\
.11\end{array}$ & $\begin{array}{r}-.32 \\
+.08 \\
.06\end{array}$ & $\begin{array}{r}0.04 \\
.26 \\
.16\end{array}$ & $\begin{array}{r}-0.34 \\
+.48 \\
.12\end{array}$ & $\begin{array}{r}0.38 \\
-.22 \\
+.04\end{array}$ \\
\hline
\end{tabular}


TABLE 6. Measured values of the deviations in meridional power at the marginal points $A$ and $B$ from the measured axial power for 71 spectacle lenses having a cylindrical power of +2.00 diopters and with spherical power varying from +7.00 to -20.00 diopters

The nominal prescribed spherical powers are listed under "Sph". The signifieance of the symbols at the heads of the columns is given in section 3.2 and figure 2. All values are expressed in diopters.

\begin{tabular}{|c|c|c|c|c|c|c|}
\hline Sph. & $\Delta V_{A}$ & $\Delta H_{A}$ & $\Delta C_{A}$ & $\Delta V_{B}$ & $\Delta H_{B}$ & $\Delta C_{B}$ \\
\hline 7.00 & $\left\{\begin{array}{r}0.14 \\
.32\end{array}\right.$ & $\begin{array}{r}-0.38 \\
-.43\end{array}$ & $\begin{array}{r}0.52 \\
.75\end{array}$ & $\begin{array}{r}-0.09 \\
-.14\end{array}$ & $\begin{array}{r}-0.33 \\
-.07\end{array}$ & $\begin{array}{r}0.24 \\
-.07\end{array}$ \\
\hline 6.00 & $\begin{array}{r}0.05 \\
.09 \\
.35 \\
.23\end{array}$ & $\begin{array}{r}-0.51 \\
-.38 \\
-.33 \\
-.41\end{array}$ & $\begin{array}{r}0.56 \\
.47 \\
.68 \\
.64\end{array}$ & $\begin{array}{r}-0.29 \\
-.17 \\
-.04 \\
-.10\end{array}$ & $\begin{array}{r}-0.20 \\
-.10 \\
+.09 \\
-.50\end{array}$ & $\begin{array}{r}-0.09 \\
-.07 \\
-.13 \\
+.40\end{array}$ \\
\hline 5.00 & $\left\{\begin{array}{r}0.04 \\
.06 \\
.15 \\
-.09\end{array}\right.$ & $\begin{array}{r}-0.37 \\
-.50 \\
-.42 \\
-.45\end{array}$ & $\begin{array}{r}0.41 \\
.56 \\
.57 \\
.36\end{array}$ & $\begin{array}{r}-0.10 \\
-.06 \\
-.09 \\
-.20\end{array}$ & $\begin{array}{r}-0.05 \\
-.10 \\
-.66 \\
-.22\end{array}$ & $\begin{array}{r}-0.05 \\
+.04 \\
.57 \\
.02\end{array}$ \\
\hline 4.00 & $\left\{\begin{array}{r}-0.15 \\
-.10 \\
+.08 \\
.09\end{array}\right.$ & $\begin{array}{r}-0.31 \\
-.38 \\
-.27 \\
-.26\end{array}$ & $\begin{array}{r}0.16 \\
.28 \\
.35 \\
.35\end{array}$ & $\begin{array}{r}-0.32 \\
-.06 \\
-.03 \\
+.02\end{array}$ & $\begin{array}{r}-0.42 \\
-.18 \\
-.01 \\
+.01\end{array}$ & $\begin{array}{r}0.10 \\
.12 \\
-.02 \\
+.01\end{array}$ \\
\hline 3.00 & $\left\{\begin{array}{r}-0.19 \\
-.33 \\
-.15 \\
-.14 \\
+.03\end{array}\right.$ & $\begin{array}{r}-0.33 \\
-.37 \\
-.35 \\
-.33 \\
-.28\end{array}$ & $\begin{array}{r}0.14 \\
.04 \\
.20 \\
.19 \\
.31\end{array}$ & $\begin{array}{r}-0.08 \\
-.08 \\
-.03 \\
-.05 \\
+.02\end{array}$ & $\begin{array}{r}-0.23 \\
-.20 \\
-.11 \\
-.15 \\
-.06\end{array}$ & $\begin{array}{l}0.15 \\
.12 \\
.08 \\
.10 \\
.08\end{array}$ \\
\hline 2. 00 & $\left\{\begin{array}{r}-0.11 \\
-.05 \\
-.02 \\
-.02 \\
-.19\end{array}\right.$ & $\begin{array}{r}-0.23 \\
-.18 \\
-.09 \\
-.19 \\
-.36\end{array}$ & $\begin{array}{r}0.12 \\
.13 \\
.07 \\
.17 \\
.17\end{array}$ & $\begin{array}{r}0.02 \\
.05 \\
-.09 \\
-.27 \\
+.01\end{array}$ & $\begin{array}{r}-0.11 \\
-.04 \\
-.29 \\
-.30 \\
-.15\end{array}$ & $\begin{array}{r}0.13 \\
.09 \\
.20 \\
.03 \\
.16\end{array}$ \\
\hline 1. 00 & $\left\{\begin{array}{r}0.02 \\
-.23 \\
+.01 \\
-.07 \\
+.08\end{array}\right.$ & $\begin{array}{r}-0.12 \\
-.22 \\
-.18 \\
-.19 \\
-.10\end{array}$ & $\begin{array}{r}0.14 \\
-.01 \\
+.18 \\
.12 \\
.18\end{array}$ & $\begin{array}{r}-0.19 \\
+.09 \\
.13 \\
.13 \\
-.14\end{array}$ & $\begin{array}{r}-0.24 \\
-.10 \\
-.05 \\
-.01 \\
-.24\end{array}$ & $\begin{array}{l}0.05 \\
.19 \\
.18 \\
.14 \\
.10\end{array}$ \\
\hline 0.00 & $\left\{\begin{array}{r}-0.11 \\
+.14 \\
.13 \\
-.13 \\
-.14\end{array}\right.$ & $\begin{array}{r}-0.14 \\
-.03 \\
-.05 \\
-.15 \\
-.16\end{array}$ & $\begin{array}{r}0.03 \\
.17 \\
.18 \\
.02 \\
.02\end{array}$ & $\begin{array}{r}+0.16 \\
-.10 \\
-.13 \\
+.13 \\
.09\end{array}$ & $\begin{array}{r}-0.04 \\
-.13 \\
-.15 \\
-.05 \\
-.08\end{array}$ & $\begin{array}{r}0.20 \\
.03 \\
.02 \\
.18 \\
.17\end{array}$ \\
\hline-1.00 & $\left\{\begin{array}{r}-0.20 \\
-.14 \\
-.10 \\
+.18 \\
.12\end{array}\right.$ & $\begin{array}{r}-0.12 \\
-.10 \\
-.09 \\
-.04 \\
-.02\end{array}$ & $\begin{array}{r}-0.08 \\
-.04 \\
-.01 \\
+.022 \\
.14\end{array}$ & $\begin{array}{r}0.16 \\
.16 \\
.15 \\
-.16 \\
-.05\end{array}$ & $\begin{array}{r}-0.03 \\
-.05 \\
-.01 \\
-.15 \\
-.11\end{array}$ & $\begin{array}{r}0.19 \\
.21 \\
.16 \\
-.01 \\
+.06\end{array}$ \\
\hline-2.00 & $\left\{\begin{array}{r}0.22 \\
-.04 \\
+.22 \\
-.11 \\
-.03\end{array}\right.$ & $\begin{array}{r}+0.01 \\
-.07 \\
+.03 \\
-.07 \\
-.09\end{array}$ & $\begin{array}{r}0.21 \\
.03 \\
.19 \\
-.04 \\
+.06\end{array}$ & $\begin{array}{r}-0.07 \\
+.23 \\
-.04 \\
+.20 \\
.21\end{array}$ & $\begin{array}{r}-0.04 \\
+.00 \\
-.09 \\
-.03 \\
+.03\end{array}$ & $\begin{array}{r}-0.03 \\
+.23 \\
.05 \\
.23 \\
.18\end{array}$ \\
\hline-3.00 & $\left\{\begin{array}{r}-0.02 \\
-.05 \\
+.02 \\
.02 \\
.04\end{array}\right.$ & $\begin{array}{r}-0.01 \\
-.04 \\
-.01 \\
-.01 \\
-.02\end{array}$ & $\begin{array}{r}-0.01 \\
-.01 \\
+.03 \\
.03 \\
.06\end{array}$ & $\begin{array}{r}0.32 \\
.22 \\
.30 \\
.26 \\
.27\end{array}$ & $\begin{array}{r}0.10 \\
.00 \\
.10 \\
.20 \\
.13\end{array}$ & $\begin{array}{r}0.22 \\
.22 \\
.20 \\
.06 \\
.14\end{array}$ \\
\hline-4.00 & $\left\{\begin{array}{r}0.02 \\
.19 \\
.32 \\
.32 \\
.02\end{array}\right.$ & $\begin{array}{r}+0.04 \\
.09 \\
.23 \\
.07 \\
.04\end{array}$ & $\begin{array}{r}-0.02 \\
+.10 \\
.09 \\
. .25 \\
-.02\end{array}$ & $\begin{array}{r}0.29 \\
.36 \\
.11 \\
.02 \\
.31\end{array}$ & $\begin{array}{r}-0.04 \\
+.25 \\
.06 \\
.04 \\
.20\end{array}$ & $\begin{array}{r}0.33 \\
.11 \\
.05 \\
-.02 \\
+.11\end{array}$ \\
\hline-6.00 & $\left\{\begin{array}{r}-0.26 \\
+.25 \\
.33 \\
.46\end{array}\right.$ & $\begin{array}{r}0.00 \\
.16 \\
.14 \\
.44\end{array}$ & $\begin{array}{r}-0.26 \\
+.09 \\
.19 \\
.02\end{array}$ & $\begin{array}{r}0.20 \\
.41 \\
.51 \\
.29\end{array}$ & $\begin{array}{r}0.15 \\
.08 \\
.31 \\
.16\end{array}$ & $\begin{array}{r}0.05 \\
.33 \\
.20 \\
.15\end{array}$ \\
\hline-10.00 & $\left\{\begin{array}{r}-0.32 \\
+.64 \\
. .91 \\
-.06\end{array}\right.$ & $\begin{array}{r}0.12 \\
.27 \\
.36 \\
.25\end{array}$ & $\begin{array}{r}-0.44 \\
+.37 \\
-.55 \\
-.31\end{array}$ & $\begin{array}{r}0.32 \\
.65 \\
.67 \\
.42\end{array}$ & $\begin{array}{r}0.20 \\
.46 \\
.45 \\
.39\end{array}$ & $\begin{array}{r}0.12 \\
.19 \\
.22 \\
.03\end{array}$ \\
\hline-12.00 & $\left\{\begin{array}{r}+0.40 \\
.44 \\
.36\end{array}\right.$ & $\begin{array}{r}0.89 \\
.41 \\
.36\end{array}$ & $\begin{array}{r}-0.49 \\
+.03 \\
.00\end{array}$ & $\begin{array}{r}0.54 \\
.11 \\
.26\end{array}$ & $\begin{array}{r}0.75 \\
.33 \\
.00\end{array}$ & $\begin{array}{r}-0.21 \\
-.22 \\
+.26\end{array}$ \\
\hline-16.00 & $\begin{array}{r}0.98 \\
.41 \\
.08\end{array}$ & $\begin{array}{r}0.66 \\
.52 \\
.26\end{array}$ & $\begin{array}{r}0.32 \\
-.11 \\
-.18\end{array}$ & $\begin{array}{r}0.68 \\
.31 \\
.26\end{array}$ & $\begin{array}{r}0.96 \\
.34 \\
-.11\end{array}$ & $\begin{array}{r}-0.28 \\
-.03 \\
+.37\end{array}$ \\
\hline-20.00 & $\left\{\begin{array}{r}0.44 \\
.37 \\
-.17\end{array}\right.$ & $\begin{array}{r}0.38 \\
.60 \\
.24\end{array}$ & $\begin{array}{r}+0.06 \\
-.23 \\
-.41\end{array}$ & $\begin{array}{r}0.62 \\
.70 \\
.02\end{array}$ & $\begin{array}{r}0.60 \\
-.39 \\
-.18\end{array}$ & $\begin{array}{r}0.02 \\
.31 \\
.20\end{array}$ \\
\hline
\end{tabular}

TABLE 7. Measured values of the deviations in meridional power at the marginal points $A$ and $B$ from the measured axial power for 43 spectacle lenses having a cylindrical power of +3.00 diopters and with spherical power varying from +6.00 to -10.00 diopters

The nominal prescribed spherical powers are listed under "Sph". The significance of the symbols at the heads of the columns is given in section 3.2 and figure 2 . All values are expressed in diopters.

\begin{tabular}{|c|c|c|c|c|c|c|}
\hline Sph. & $\Delta V_{A}$ & $\Delta H_{A}$ & $\Delta C_{A}$ & $\Delta V_{B}$ & $\Delta H_{B}$ & $\Delta C_{B}$ \\
\hline 6.00 & $\left\{\begin{array}{r}0.32 \\
.37 \\
.30 \\
.64\end{array}\right.$ & $\begin{array}{r}-0.53 \\
-.57 \\
-.47 \\
-.46\end{array}$ & $\begin{array}{r}0.85 \\
.94 \\
.77 \\
1.10\end{array}$ & $\begin{array}{r}-0.05 \\
-.18 \\
-.15 \\
+.13\end{array}$ & $\begin{array}{r}0.07 \\
-.12 \\
-.08 \\
+.19\end{array}$ & $\begin{array}{r}-0.12 \\
-.06 \\
-.07 \\
-.06\end{array}$ \\
\hline 5.00 & $\left\{\begin{array}{r}-0.03 \\
+.19 \\
.57 \\
.18\end{array}\right.$ & $\begin{array}{r}-0.54 \\
-.46 \\
-.40 \\
-.53\end{array}$ & $\begin{array}{r}0.51 \\
.65 \\
.97 \\
.71\end{array}$ & $\begin{array}{r}-0.21 \\
-.07 \\
+.10 \\
-.06\end{array}$ & $\begin{array}{r}-0.30 \\
-.13 \\
+.12 \\
-.57\end{array}$ & $\begin{array}{r}+0.09 \\
.06 \\
-.02 \\
+.51\end{array}$ \\
\hline 4. 00 & $\left\{\begin{array}{r}-0.09 \\
+.12 \\
.30 \\
-.17\end{array}\right.$ & $\begin{array}{r}-0.50 \\
-.30 \\
-.47 \\
-.53\end{array}$ & $\begin{array}{l}0.41 \\
.42 \\
.77 \\
.36\end{array}$ & $\begin{array}{r}-0.11 \\
+.07 \\
-.00 \\
-.18\end{array}$ & $\begin{array}{r}-0.22 \\
+.04 \\
-.13 \\
-.40\end{array}$ & $\begin{array}{r}0.11 \\
.03 \\
.13 \\
.22\end{array}$ \\
\hline 3.00 & $\left\{\begin{array}{r}-0.13 \\
-.08 \\
-.33 \\
-.17\end{array}\right.$ & $\begin{array}{r}-0.37 \\
-.48 \\
-.47 \\
-.34\end{array}$ & $\begin{array}{r}0.24 \\
.40 \\
.14 \\
.17\end{array}$ & $\begin{array}{r}+0.04 \\
-.03 \\
+.03 \\
-.02\end{array}$ & $\begin{array}{r}-0.05 \\
-.14 \\
-.15 \\
-.09\end{array}$ & $\begin{array}{r}0.09 \\
.11 \\
.18 \\
.07\end{array}$ \\
\hline-1.00 & $\left\{\begin{array}{r}-0.19 \\
-.29 \\
-.25 \\
-.21 \\
-.24\end{array}\right.$ & $\begin{array}{r}-0.17 \\
-.19 \\
-.25 \\
-.19 \\
-.20\end{array}$ & $\begin{array}{r}-0.02 \\
-.10 \\
-.00 \\
-.02 \\
-.04\end{array}$ & $\begin{array}{r}+0.22 \\
.23 \\
.14 \\
.24 \\
.22\end{array}$ & $\begin{array}{r}+0.01 \\
-.04 \\
-.01 \\
-.10 \\
-.01\end{array}$ & $\begin{array}{r}0.21 \\
.27 \\
.15 \\
.34 \\
.23\end{array}$ \\
\hline-2.00 & $\left\{\begin{array}{r}-0.24 \\
-.20 \\
-.09 \\
-.08 \\
-.33\end{array}\right.$ & $\begin{array}{r}-0.15 \\
-.16 \\
-.17 \\
-.17 \\
-.30\end{array}$ & $\begin{array}{r}-0.09 \\
-.04 \\
+.08 \\
.09 \\
-.03\end{array}$ & $\begin{array}{r}0.27 \\
.34 \\
.25 \\
.34 \\
.18\end{array}$ & $\begin{array}{r}+0.04 \\
.03 \\
-.02 \\
+.11 \\
-.15\end{array}$ & $\begin{array}{r}0.23 \\
.31 \\
.27 \\
.23 \\
.33\end{array}$ \\
\hline-3.00 & $\left\{\begin{array}{r}-0.03 \\
-.12 \\
-.10 \\
-.11 \\
-.12\end{array}\right.$ & $\begin{array}{r}-0.06 \\
-.14 \\
-.13 \\
-.12 \\
-.13\end{array}$ & $\begin{array}{r}+0.03 \\
.02 \\
.03 \\
.01 \\
.01\end{array}$ & $\begin{array}{r}0.34 \\
.39 \\
.35 \\
.38 \\
.36\end{array}$ & $\begin{array}{r}-0.04 \\
+.03 \\
.07 \\
.02 \\
.11\end{array}$ & $\begin{array}{r}0.38 \\
.36 \\
.28 \\
.36 \\
.25\end{array}$ \\
\hline-4.00 & $\left\{\begin{array}{r}0.00 \\
-.03 \\
-.07 \\
-.05\end{array}\right.$ & $\begin{array}{r}-0.05 \\
-.05 \\
-.08 \\
-.05\end{array}$ & $\begin{array}{r}0.05 \\
.02 \\
.01 \\
.00\end{array}$ & $\begin{array}{r}0.49 \\
.40 \\
.41 \\
.40\end{array}$ & $\begin{array}{r}0.26 \\
-.01 \\
+.10 \\
.30\end{array}$ & $\begin{array}{r}0.23 \\
.41 \\
.31 \\
.10\end{array}$ \\
\hline-6.00 & $\left\{\begin{array}{r}+0.46 \\
.30 \\
-.14 \\
+.21\end{array}\right.$ & $\begin{array}{r}+0.14 \\
.11 \\
-.07 \\
+.10\end{array}$ & $\begin{array}{r}0.32 \\
.19 \\
-.07 \\
+.11\end{array}$ & $\begin{array}{r}0.71 \\
.59 \\
.36 \\
.55\end{array}$ & $\begin{array}{r}0.38 \\
.48 \\
.21 \\
.06\end{array}$ & $\begin{array}{r}0.33 \\
.11 \\
.15 \\
.49\end{array}$ \\
\hline-10.00 & $\left\{\begin{array}{r}0.01 \\
-.22 \\
+.71 \\
.81\end{array}\right.$ & $\begin{array}{r}0.35 \\
.06 \\
.14 \\
.23\end{array}$ & $\begin{array}{r}-0.34 \\
-.28 \\
+.57 \\
.58\end{array}$ & $\begin{array}{r}0.57 \\
.33 \\
.78 \\
.85\end{array}$ & $\begin{array}{r}0.64 \\
.40 \\
.34 \\
.86\end{array}$ & $\begin{array}{r}-0.07 \\
-.07 \\
+.44 \\
-.01\end{array}$ \\
\hline
\end{tabular}

TABLE 8. Measured values of the deviations in the meridional power at marginal points $A$ and $B$ from the measured axial power for 8 spectacle lenses having a cylindrical power of +5.00 diopters and with spherical powers of -1.00 and -2.00 diopters

All powers are expressed in diopters

\begin{tabular}{|c|c|c|c|c|c|c|}
\hline Sph. & $\Delta V_{A}$ & $\Delta H_{A}$ & $\Delta C_{A}$ & $\Delta V_{B}$ & $\Delta H_{B}$ & $\Delta C_{B}$ \\
\hline-1.00 & $\left\{\begin{array}{r}-0.21 \\
-.43 \\
-.38 \\
-.33\end{array}\right.$ & $\begin{array}{r}-0.43 \\
-.43 \\
-.41 \\
-.31\end{array}$ & $\begin{array}{r}0.22 \\
.00 \\
.03 \\
-.02\end{array}$ & $\begin{array}{r}0.43 \\
.28 \\
.39 \\
.34\end{array}$ & $\begin{array}{r}-0.04 \\
-.11 \\
-.08 \\
-.04\end{array}$ & $\begin{array}{r}0.47 \\
.39 \\
.47 \\
.38\end{array}$ \\
\hline-2.00 & $\left\{\begin{array}{r}-0.26 \\
-.36 \\
-.42 \\
-.27\end{array}\right.$ & $\begin{array}{r}-0.34 \\
-.36 \\
-.34 \\
-.29\end{array}$ & $\begin{array}{r}+0.08 \\
.00 \\
-.08 \\
+.02\end{array}$ & $\begin{array}{r}0.56 \\
.26 \\
.48 \\
.45\end{array}$ & $\begin{array}{r}+0.01 \\
-.05 \\
+.04 \\
.10\end{array}$ & $\begin{array}{r}0.55 \\
.31 \\
.44 \\
.35\end{array}$ \\
\hline
\end{tabular}


TABLE 9. Measured values of the deviations in meridional power at the marginal points $A$ and $B$ from the measured axial power for 33 spectacle lenses having a cylindrical power of +4.00 diopters and with spherical power varying from +5.00 to -10.00 diopters

The nominal prescribed spherical powers are listed under "Sph." The significance of the symbols at the heads of the columns is given in section 3.2 and figure 2 . All values are expressed in diopters.

\begin{tabular}{|c|c|c|c|c|c|c|}
\hline Sph. & $\Delta V_{A}$ & $\Delta H_{A}$ & $\Delta C_{A}$ & $\Delta V_{B}$ & $\Delta H_{B}$ & $\Delta C_{B}$ \\
\hline 5. 00 & $\begin{array}{r}0.24 \\
-.03 \\
+.32 \\
.25\end{array}$ & $\begin{array}{r}-0.61 \\
-.74 \\
-.56 \\
-.75\end{array}$ & $\begin{array}{r}0.85 \\
.71 \\
.88 \\
1.00\end{array}$ & $\begin{array}{r}0.01 \\
-.19 \\
+.00 \\
-.01\end{array}$ & $\begin{array}{r}-0.30 \\
-.27 \\
-.07 \\
-.05\end{array}$ & $\begin{array}{r}0.31 \\
.08 \\
.07 \\
.04\end{array}$ \\
\hline 4. 00 & $\left\{\begin{array}{r}-0.09 \\
-.09 \\
+.28 \\
.12\end{array}\right.$ & $\begin{array}{r}-0.65 \\
-.57 \\
-.53 \\
-.54\end{array}$ & $\begin{array}{r}0.56 \\
.48 \\
.81 \\
.66\end{array}$ & $\begin{array}{r}-0.11 \\
-.03 \\
+.18 \\
.01\end{array}$ & $\begin{array}{r}-0.41 \\
-.25 \\
+.04 \\
-.11\end{array}$ & $\begin{array}{r}0.30 \\
.22 \\
.14 \\
.12\end{array}$ \\
\hline-1.00 & $\left\{\begin{array}{r}-0.33 \\
-.29 \\
-.35 \\
-.16\end{array}\right.$ & $\begin{array}{r}-0.33 \\
-.29 \\
-.31 \\
-.29\end{array}$ & $\begin{array}{r}0.00 \\
.00 \\
-.04 \\
+.13\end{array}$ & $\begin{array}{r}0.23 \\
.30 \\
.23 \\
.38\end{array}$ & $\begin{array}{r}-0.02 \\
-.04 \\
-.10 \\
-.04\end{array}$ & $\begin{array}{r}0.25 \\
.34 \\
.33 \\
.42\end{array}$ \\
\hline-2.00 & $\left\{\begin{array}{r}-0.24 \\
-.35 \\
-.27 \\
-.23\end{array}\right.$ & $\begin{array}{r}-0.29 \\
-.27 \\
-.25 \\
-.25\end{array}$ & $\begin{array}{r}0.05 \\
-.08 \\
-.02 \\
+.02\end{array}$ & $\begin{array}{r}0.31 \\
.35 \\
.32 \\
.32\end{array}$ & $\begin{array}{r}+0.07 \\
.01 \\
.05 \\
.01\end{array}$ & $\begin{array}{r}0.24 \\
.34 \\
.27 \\
.31\end{array}$ \\
\hline-3.00 & $\left\{\begin{array}{r}-0.23 \\
-.17 \\
-.41 \\
-.23\end{array}\right.$ & $\begin{array}{r}-0.19 \\
-.19 \\
-.20 \\
-.24\end{array}$ & $\begin{array}{r}-0.04 \\
+.02 \\
-.21 \\
+.01\end{array}$ & $\begin{array}{r}0.42 \\
.43 \\
.43 \\
.39\end{array}$ & $\begin{array}{r}0.02 \\
.03 \\
.08 \\
.16\end{array}$ & $\begin{array}{r}0.40 \\
.40 \\
.35 \\
.23\end{array}$ \\
\hline-4.00 & $\left\{\begin{array}{r}-0.10 \\
-.04 \\
-.09 \\
-.08 \\
-.08\end{array}\right.$ & $\begin{array}{r}-0.16 \\
-.07 \\
-.13 \\
-.10 \\
-.15\end{array}$ & $\begin{array}{l}0.06 \\
.03 \\
.04 \\
.02 \\
.07\end{array}$ & $\begin{array}{r}0.50 \\
.48 \\
.57 \\
.47 \\
.51\end{array}$ & $\begin{array}{r}0.27 \\
-.02 \\
+.14 \\
-.01 \\
+.22\end{array}$ & $\begin{array}{r}0.23 \\
.50 \\
.43 \\
.48 \\
.29\end{array}$ \\
\hline-6.00 & $\left\{\begin{array}{r}+0.06 \\
.18 \\
.06 \\
-.22\end{array}\right.$ & $\begin{array}{r}-0.03 \\
+.02 \\
-.03 \\
-.23\end{array}$ & $\begin{array}{r}0.09 \\
.16 \\
.09 \\
.01\end{array}$ & $\begin{array}{r}0.60 \\
.75 \\
.57 \\
.38\end{array}$ & $\begin{array}{r}0.00 \\
.26 \\
.39 \\
.42\end{array}$ & $\begin{array}{r}0.60 \\
.49 \\
.18 \\
-.04\end{array}$ \\
\hline-10.00 & $\left\{\begin{array}{r}+0.84 \\
-.21 \\
-.32 \\
+.65\end{array}\right.$ & $\begin{array}{r}+0.25 \\
.24 \\
.09 \\
.23\end{array}$ & $\begin{array}{r}0.59 \\
-.45 \\
-.41 \\
+.42\end{array}$ & $\begin{array}{r}1.09 \\
.59 \\
.42 \\
.95\end{array}$ & $\begin{array}{r}1.01 \\
0.63 \\
.35 \\
.51\end{array}$ & $\begin{array}{r}+0.08 \\
-.04 \\
+.07 \\
.44\end{array}$ \\
\hline
\end{tabular}

TABLE 10. Measured values of the deviations in the meridional power at marginal points $A$ and $B$ from the measured axial power for 8 spectacle lenses having a cylindrical power of +6.00 diopters and with spherical powers of -1.00 and -2.00 diopters

All powers are expressed in diopters

\begin{tabular}{|c|c|c|c|c|c|c|}
\hline Sph. & $\Delta V_{A}$ & $\Delta H_{A}$ & $\Delta C_{A}$ & $\Delta V_{B}$ & $\Delta H_{B}$ & $\Delta C_{B}$ \\
\hline-1.00 & $\left\{\begin{array}{r}-0.45 \\
-.29 \\
-.37 \\
-.33\end{array}\right.$ & $\begin{array}{r}-0.49 \\
-.20 \\
-.51 \\
-.34\end{array}$ & $\begin{array}{r}0.04 \\
-.09 \\
+.14 \\
.01\end{array}$ & $\begin{array}{r}0.32 \\
.53 \\
.31 \\
.47\end{array}$ & $\begin{array}{r}-0.15 \\
-.06 \\
-.08 \\
+.02\end{array}$ & $\begin{array}{r}0.47 \\
.59 \\
.39 \\
.45\end{array}$ \\
\hline-2.00 & $\left\{\begin{array}{r}-0.39 \\
-.32 \\
-.47 \\
-.46\end{array}\right.$ & $\begin{array}{r}-0.36 \\
-.35 \\
-.48 \\
-.47\end{array}$ & $\begin{array}{r}-0.03 \\
+.03 \\
.01 \\
.01\end{array}$ & $\begin{array}{r}0.44 \\
.65 \\
.40 \\
.51\end{array}$ & $\begin{array}{r}0.04 \\
.01 \\
-.02 \\
+.02\end{array}$ & $\begin{array}{r}0.40 \\
.64 \\
.42 \\
.49\end{array}$ \\
\hline
\end{tabular}

(c) The tolerances in marginal power contained in paragraphs (a) and (b) are only applicable to single vision lenses and to the distance portions of bifocal lenses. The range for which these tolerances apply is as shown in tables 11 to 15 . The tolerances shown in tables 11 to 15 apply to lenses corrected for optimum marginal performance at large object distances. For lenses corrected for optimum marginal performance at near distances, the same toler-
TABLE 11. Tolerances in meridional and cylindrical powers at $30^{\circ}$ from the axis for lenses having spherical powers ranging from +7.00 to -20.00 diopters and zero cylindrical power

$V_{O}$ is the measured axial meridional power in one principal meridian and $H_{0}$ is the measured axial meridional power in the second principal meridian $\left(H_{O}=V_{O}\right.$ and corresponds to the prescribed spherical power.)

\begin{tabular}{|c|c|c|c|c|}
\hline \multirow{2}{*}{$\begin{array}{c}\text { Meridional } \\
\text { powers } \\
\left(V_{O}=H_{O}\right)\end{array}$} & \multicolumn{2}{|c|}{ Meridional power } & \multicolumn{2}{|c|}{ Cylindrical power } \\
\hline & $\begin{array}{l}\text { Point } A \\
\text { and } \\
\text { point } B\end{array}$ & $\begin{array}{l}\text { Point } A \\
\text { or } \\
\text { point } B\end{array}$ & $\begin{array}{l}\text { Point } A \\
\text { and } \\
\text { point } B\end{array}$ & $\begin{array}{c}\text { Point } A \\
\text { or } \\
\text { point } B\end{array}$ \\
\hline $\begin{array}{l}7.00 \\
6.00 \\
5.00 \\
4.00 \\
\text { 3. } 00\end{array}$ & $\begin{array}{r} \pm 0.38 \\
.38 \\
.32 \\
.32 \\
.25\end{array}$ & $\begin{array}{r} \pm 0.32 \\
.32 \\
.25 \\
.25 \\
.18\end{array}$ & $\begin{array}{r} \pm 0.38 \\
.32 \\
.32 \\
.25 \\
.18\end{array}$ & $\begin{array}{r} \pm 0.32 \\
.25 \\
.25 \\
.18 \\
.12\end{array}$ \\
\hline $\begin{array}{r}2.00 \\
1.00 \\
0.00 \\
-1.00 \\
-2.00\end{array}$ & $\begin{array}{r} \pm 0.18 \\
.18 \\
.18 \\
.18 \\
.25\end{array}$ & $\begin{array}{r} \pm 0.12 \\
.12 \\
.12 \\
.12 \\
.18\end{array}$ & $\begin{array}{r} \pm 0.18 \\
.18 \\
.18 \\
.18 \\
.18\end{array}$ & $\begin{array}{r} \pm 0.12 \\
.12 \\
.12 \\
.12 \\
.12\end{array}$ \\
\hline $\begin{array}{l}-3.00 \\
-4.00 \\
-5.00 \\
-6.00 \\
-7.00\end{array}$ & $\begin{array}{r} \pm 0.25 \\
.32 \\
.32 \\
.38 \\
.38\end{array}$ & $\begin{array}{r} \pm 0.18 \\
.25 \\
.25 \\
.32 \\
.32\end{array}$ & $\begin{array}{r} \pm 0.18 \\
.25 \\
.32 \\
.32 \\
.32\end{array}$ & $\begin{array}{r} \pm 0.12 \\
.18 \\
.25 \\
.25 \\
.25\end{array}$ \\
\hline $\begin{array}{r}-8.00 \\
-9.00 \\
-10.00 \\
-14.00 \\
-20.00\end{array}$ & $\begin{array}{r} \pm 0.38 \\
.50 \\
.50 \\
.50 \\
.50\end{array}$ & $\begin{array}{r} \pm 0.32 \\
.38 \\
.38 \\
.38 \\
.38\end{array}$ & $\begin{array}{r} \pm 0.32 \\
.38 \\
.38 \\
.38 \\
.38\end{array}$ & $\begin{array}{r} \pm 0.25 \\
.32 \\
.32 \\
.32 \\
.32\end{array}$ \\
\hline
\end{tabular}

TABLE 12. Tolerances in meridional and cylindrical powers for lenses having spherical powers ranging from +7.00 to -20.00 diopters and a cylindrical power of 1.00 diopter

$V_{O}$ is the measured axial meridional power in one principal meridian and $H_{0}$ is the measured axial meridional power in the second principal meridian. (For positive cylindrical power, $H_{0}$ corresponds to the premeridian. responds to the prescribed spherical power.) All values are given in diopters.

\begin{tabular}{|c|c|c|c|c|c|}
\hline \multicolumn{2}{|c|}{ Meridional powers } & \multicolumn{2}{|c|}{ Meridional power } & \multicolumn{2}{|c|}{ Cylindrical power } \\
\hline$V_{o}$ & $H_{O}$ & $\begin{array}{l}\text { Point } A \\
\text { and } \\
\text { point } B\end{array}$ & $\begin{array}{c}\text { Point } A \\
\text { or } \\
\text { point } B\end{array}$ & $\begin{array}{l}\text { Point } A \\
\text { and } \\
\text { point } B\end{array}$ & $\begin{array}{c}\text { Point } A \\
\text { or } \\
\text { point } B\end{array}$ \\
\hline $\begin{array}{l}8.00 \\
7.00 \\
6.00 \\
5.00 \\
4.00\end{array}$ & $\begin{array}{l}\text { 7. } 00 \\
6.00 \\
5.00 \\
\text { 4. } 00 \\
\text { 3. } 00\end{array}$ & $\begin{array}{r} \pm 0.62 \\
.50 \\
.50 \\
.38 \\
.38\end{array}$ & $\begin{array}{r} \pm 0.50 \\
.38 \\
.38 \\
.25 \\
.25\end{array}$ & $\begin{array}{r} \pm 0.50 \\
.50 \\
.38 \\
.32 \\
.25\end{array}$ & $\begin{array}{r} \pm 0.38 \\
.38 \\
.25 \\
.25 \\
.18\end{array}$ \\
\hline $\begin{array}{r}3.00 \\
2.00 \\
1.00 \\
0.00 \\
-1.00\end{array}$ & $\begin{array}{r}2.00 \\
1.00 \\
0.00 \\
-1.00 \\
-2.00\end{array}$ & $\begin{array}{r} \pm 0.25 \\
.18 \\
.18 \\
.18 \\
.25\end{array}$ & $\begin{array}{r} \pm 0.18 \\
.12 \\
.12 \\
12 \\
.18\end{array}$ & $\begin{array}{r} \pm 0.18 \\
.18 \\
.18 \\
.18 \\
.18\end{array}$ & $\begin{array}{r} \pm 0.12 \\
.12 \\
.12 \\
.12 \\
.12\end{array}$ \\
\hline $\begin{array}{l}-2.00 \\
-3.00 \\
-4.00 \\
-5.00 \\
-6.00\end{array}$ & $\begin{array}{l}-3.00 \\
-4.00 \\
-5.00 \\
-6.00 \\
-7.00\end{array}$ & $\begin{array}{r} \pm 0.38 \\
.38 \\
.50 \\
.50 \\
.50\end{array}$ & $\begin{array}{r} \pm 0.25 \\
.25 \\
.38 \\
.38 \\
.38\end{array}$ & $\begin{array}{r} \pm 0.25 \\
.32 \\
.38 \\
.38 \\
.38\end{array}$ & $\begin{array}{r} \pm 0.18 \\
.25 \\
.25 \\
.25 \\
.25\end{array}$ \\
\hline $\begin{array}{r}-7.00 \\
-8.00 \\
-9.00 \\
-14.00 \\
-19.00\end{array}$ & $\begin{array}{r}-8.00 \\
-9.00 \\
-10.00 \\
-15.00 \\
-20.00\end{array}$ & $\begin{array}{r} \pm 0.50 \\
.62 \\
.62 \\
.62 \\
.62\end{array}$ & $\begin{array}{r} \pm 0.38 \\
.38 \\
.50 \\
.50 \\
.50\end{array}$ & $\begin{array}{r} \pm 0.38 \\
.50 \\
.50 \\
.50 \\
.50\end{array}$ & $\begin{array}{r} \pm 0.25 \\
.38 \\
.38 \\
.38 \\
.38\end{array}$ \\
\hline
\end{tabular}

ances shall apply, except that the measurements will be made with the test object at the specified near object distance, and the tolerances will be referred to the measured effective axial meridional and cylindrical powers. 
TABLE 13. Tolerances in meridional and cylindrical powers for lenses having spherical powers ranging from +7.00 to -20.00 diopters and a cylindrical pow er of 2.00 diopters

$V_{O}$ is the measured axial meridional power in one principal meridian and $\mathrm{Ho}_{\mathrm{O}}$ is the measured axial meridional power in the second principal meridian. (For positive cylindrical power, $H_{0}$ corresponds to the prescribed spherical power, while for negative cylindrical power, $V 0$ corresponds to the preseribed spherical power ) All values are given in diopters.

\begin{tabular}{|c|c|c|c|c|c|}
\hline \multicolumn{2}{|c|}{ Meridional powers } & \multicolumn{2}{|c|}{ Meridional power } & \multicolumn{2}{|c|}{ Cylindrical power } \\
\hline$V_{O}$ & $H_{o}$ & $\begin{array}{l}\text { Point } A \\
\text { and } \\
\text { point } B\end{array}$ & $\begin{array}{l}\text { Point } A \\
\text { or } \\
\text { point } B\end{array}$ & $\begin{array}{l}\text { Point } A \\
\text { and } \\
\text { point } B\end{array}$ & $\begin{array}{l}\text { Point } A \\
\text { or } \\
\text { point } B\end{array}$ \\
\hline $\begin{array}{l}9.00 \\
8.00 \\
7.00 \\
6.00 \\
5.00\end{array}$ & $\begin{array}{l}7.00 \\
6.00 \\
5.00 \\
4.00 \\
3.00\end{array}$ & $\begin{array}{r} \pm 0.62 \\
.62 \\
.50 \\
.38 \\
.38\end{array}$ & $\begin{array}{r} \pm 0.50 \\
.50 \\
.38 \\
.25 \\
.25\end{array}$ & $\begin{array}{r} \pm 0.62 \\
.62 \\
.50 \\
.38 \\
.32\end{array}$ & $\begin{array}{r} \pm 0.50 \\
.38 \\
.25 \\
.25 \\
.25\end{array}$ \\
\hline $\begin{array}{l}4.00 \\
3.00 \\
2.00 \\
1.00 \\
0.00\end{array}$ & $\begin{array}{r}2.00 \\
1.00 \\
0.00 \\
-1.00 \\
-2.00\end{array}$ & $\begin{array}{r} \pm 0.38 \\
.38 \\
.38 \\
.25 \\
.25\end{array}$ & $\begin{array}{r} \pm 0.25 \\
.25 \\
.25 \\
.25 \\
.25\end{array}$ & $\begin{array}{r} \pm 0.25 \\
.25 \\
.25 \\
.25 \\
.25\end{array}$ & $\begin{array}{r} \pm 0.18 \\
.18 \\
.18 \\
.18 \\
.18\end{array}$ \\
\hline $\begin{array}{l}-1.00 \\
-2.00 \\
-3.00 \\
-4.00 \\
-5.00\end{array}$ & $\begin{array}{l}-3.00 \\
-4.00 \\
-5.00 \\
-6.00 \\
-7.00\end{array}$ & $\begin{array}{r} \pm 0.38 \\
.38 \\
.50 \\
.62 \\
.62\end{array}$ & $\begin{array}{r} \pm 0.25 \\
.25 \\
.38 \\
.50 \\
.50\end{array}$ & $\begin{array}{r} \pm 0.32 \\
.38 \\
.38 \\
.50 \\
.62\end{array}$ & $\begin{array}{r} \pm 0.25 \\
.25 \\
.32 \\
.38 \\
.50\end{array}$ \\
\hline $\begin{array}{r}-6.00 \\
-7.00 \\
-8.00 \\
-13.00 \\
-18.00\end{array}$ & $\begin{array}{r}-8.00 \\
-9.00 \\
-10.00 \\
-15.00 \\
-20.00\end{array}$ & $\begin{array}{r} \pm 0.62 \\
.62 \\
.62 \\
.62 \\
.62\end{array}$ & $\begin{array}{r} \pm 0.50 \\
.50 \\
.50 \\
.50 \\
.50\end{array}$ & $\begin{array}{r} \pm 0.62 \\
.62 \\
.62 \\
.62 \\
.62\end{array}$ & $\begin{array}{r} \pm 0.50 \\
.50 \\
.50 \\
.50 \\
.50\end{array}$ \\
\hline
\end{tabular}

TABLE 14. Tolerances in meridional and cylindrical powers for lenses having spherical powers ranging from +6.00 to -10.00 diopters and a cylindrical power of 3.00 diopters.

$V_{O}$ is the measured axial meridional power in one principal meridian and $H_{O}$ is the measured axial meridional power in the second principal meridian. (For positive cylindrical power, $H_{O}$ corresponds to the prescribed spherical power, while for negative cylindrical power, $V_{0}$ corresponds to the prescribed spherical power.) All values are given in diopters.

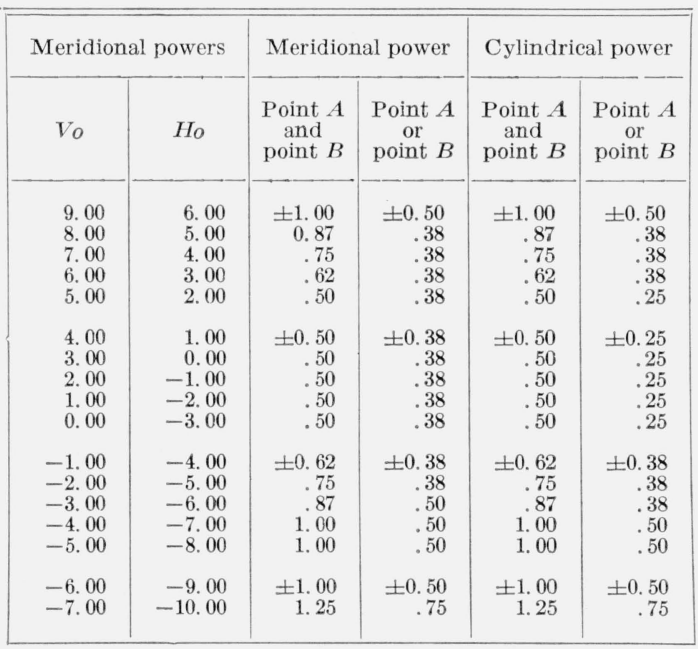

Lenses, having cylindrical power, differ in meridional power in their two principal meridians. This introduces a duality that is reflected in the setting of tolerances. It is obvious that a tolerance that is entirely satisfactory for the lower ot the two meridional powers may be too rigorous for the higher one. Likewise, a tolerance that is suitable for the higher of the two meridional powers may be less exacting than is desirable for the lower one. Conse-
TABLE 15. Tolerances in meridional and cylindrical powers for lenses having spherical powers ranging from +6.00 to - 10.00 diopters and a cylindrical power of 4.00 diopters.

$V o$ is the measured axial meridional power in one principal meridian and $H_{o}$ is the measured axial meridional power in the second principal meridian. (For positive cylindrical power, $H_{0}$ corresponds to the prescribed spherical power power.) All values are given in diopters.

\begin{tabular}{|c|c|c|c|c|c|}
\hline \multicolumn{2}{|c|}{ Meridional powers } & \multicolumn{2}{|c|}{ Meridional power } & \multicolumn{2}{|c|}{ Cylindrical power } \\
\hline$V_{0}$ & $H_{0}$ & $\begin{array}{l}\text { Point } A \\
\text { and } \\
\text { point } B\end{array}$ & $\begin{array}{l}\text { Point } A \\
\text { or } \\
\text { point } B\end{array}$ & $\begin{array}{l}\text { Point } A \\
\text { and } \\
\text { point } B\end{array}$ & $\begin{array}{l}\text { Point } A \\
\text { or } \\
\text { point } B\end{array}$ \\
\hline $\begin{array}{r}10.00 \\
9.00 \\
8.00 \\
7.00 \\
6.00\end{array}$ & $\begin{array}{l}6.00 \\
5.00 \\
4.00 \\
3.00 \\
2.00\end{array}$ & $\begin{array}{r} \pm 1.00 \\
.87 \\
.75 \\
.62 \\
.50\end{array}$ & $\begin{array}{r} \pm 0.50 \\
.38 \\
.38 \\
.38 \\
.38\end{array}$ & $\begin{array}{r} \pm 1.00 \\
.87 \\
.75 \\
.62 \\
.50\end{array}$ & $\begin{array}{r} \pm 0.50 \\
.38 \\
.38 \\
.38 \\
.25\end{array}$ \\
\hline $\begin{array}{l}5.00 \\
4.00 \\
3.00 \\
2.00 \\
1.00\end{array}$ & $\begin{array}{r}1.00 \\
0.00 \\
-1.00 \\
-2.00 \\
-3.00\end{array}$ & $\begin{array}{r} \pm 0.50 \\
.50 \\
.50 \\
.50 \\
.62\end{array}$ & $\begin{array}{r} \pm 0.38 \\
.38 \\
.38 \\
.38 \\
.38\end{array}$ & $\begin{array}{r} \pm 0.50 \\
.50 \\
.50 \\
.50 \\
.62\end{array}$ & $\begin{array}{r} \pm 0.25 \\
.25 \\
.25 \\
.25 \\
.38\end{array}$ \\
\hline $\begin{array}{r}0.00 \\
-1.00 \\
-2.00 \\
-3.00 \\
-4.00\end{array}$ & $\begin{array}{l}-4.00 \\
-5.00 \\
-6.00 \\
-7.00 \\
-8.00\end{array}$ & $\begin{array}{r} \pm 0.75 \\
.87 \\
1.00 \\
1.00 \\
1.00\end{array}$ & $\begin{array}{r} \pm 0.38 \\
.38 \\
.50 \\
.50 \\
.50\end{array}$ & $\begin{array}{r} \pm 0.75 \\
.87 \\
1.00 \\
1.00 \\
1.00\end{array}$ & $\begin{array}{r} \pm 0.38 \\
.38 \\
.50 \\
.50 \\
.50\end{array}$ \\
\hline $\begin{array}{l}-5.00 \\
-6.00\end{array}$ & $\begin{array}{r}-9.00 \\
-10.00\end{array}$ & $\begin{array}{r} \pm 1.00 \\
1.25\end{array}$ & $\begin{array}{r} \pm 0.50 \\
.75\end{array}$ & $\begin{array}{r} \pm 1.00 \\
1.25\end{array}$ & $\begin{array}{r} \pm 0.50 \\
.75\end{array}$ \\
\hline
\end{tabular}

quently, in tables 11 to 15 , one tolerance is listed that must be satisfied at both points $A$ and $B$. Assuming that a given lens satisfies the large tolerance established for both points $A$ and $B$, then for acceptance, it must also satisfy a lower value of the tolerance for either point $A$ or point $B$. It is evident that the dual tolerances are necessary in tables 12 to 15 , which list the tolerances for lenses having cylindrical power. In the case of lenses having no nominal cylindrical power, the presence of small amounts of tolerable axial cylindrical power necessitates a dual listing of tolerance in table 11.

\subsection{Degree of Compliance With Proposed Tolerances}

The results of the measurements of marginal power made on the 311 lenses that are contained in tables 4 to 10 have been considered with respect to these tolerances to determine the degree of compliance. For the cases of lenses having axial cylindrical powers of $0.00,1.00$, and 2.00 , the results of these considerations are shown graphically in figures 3 to 5 . In the graphs, the abscissas show the values of axial meridional power, $V_{o}$, while the erdinates show the deviations in marginal meridional power and in cylindrical power at points $A$ and $B$. The dotted lines bounding the horizontal zone in the central regions of the graph indicate the maximum tolerances for marginal performance for both $A$ and $B$. The solid lines bound the zone of minimum tolerance for either $A$ or $B$. To avoid plotting a multiplicity of points, only those are shown where one or both of the points fall outside tolerance. The uppermost frame of a given graph shows the nature of the lack of compliance with the meridional requirements for point $A$, the middle frame shows similar information 


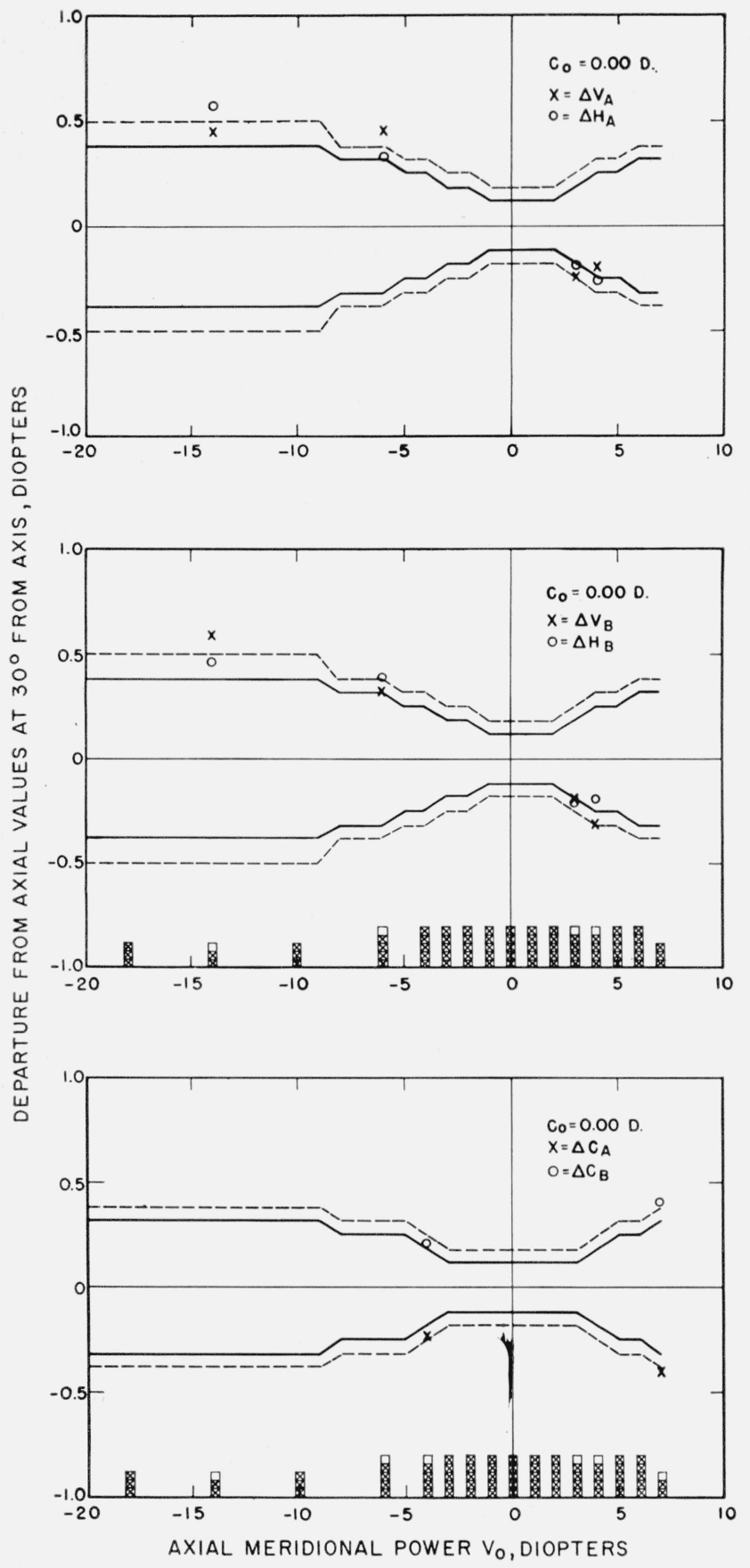

Figure 3. Degree of compliance with suggested tolerance for marginal power for 72 lenses having zero cylindrical power.

Only those points are plotted for which noncompliance occurs for at least one member of a pair. The bar graphs at the bottoms of the center and lower frames show the number of lenses in the sample. Boxes containing an $\mathrm{X}$ in the center frame show compliance with the spherical tolerances; boxes containing an $\mathrm{X}$ in the lower frame indicate full compliance with spherical and cylindrical tolerances.

for point $B$, and in addition the boxes in the bar graph in the lower portion shows the number of lenses in each sample. The number of boxes containing an $X$ is the number of lenses of a given power that comply with the meridional requirements. The empty boxes indicate those lenses where at least
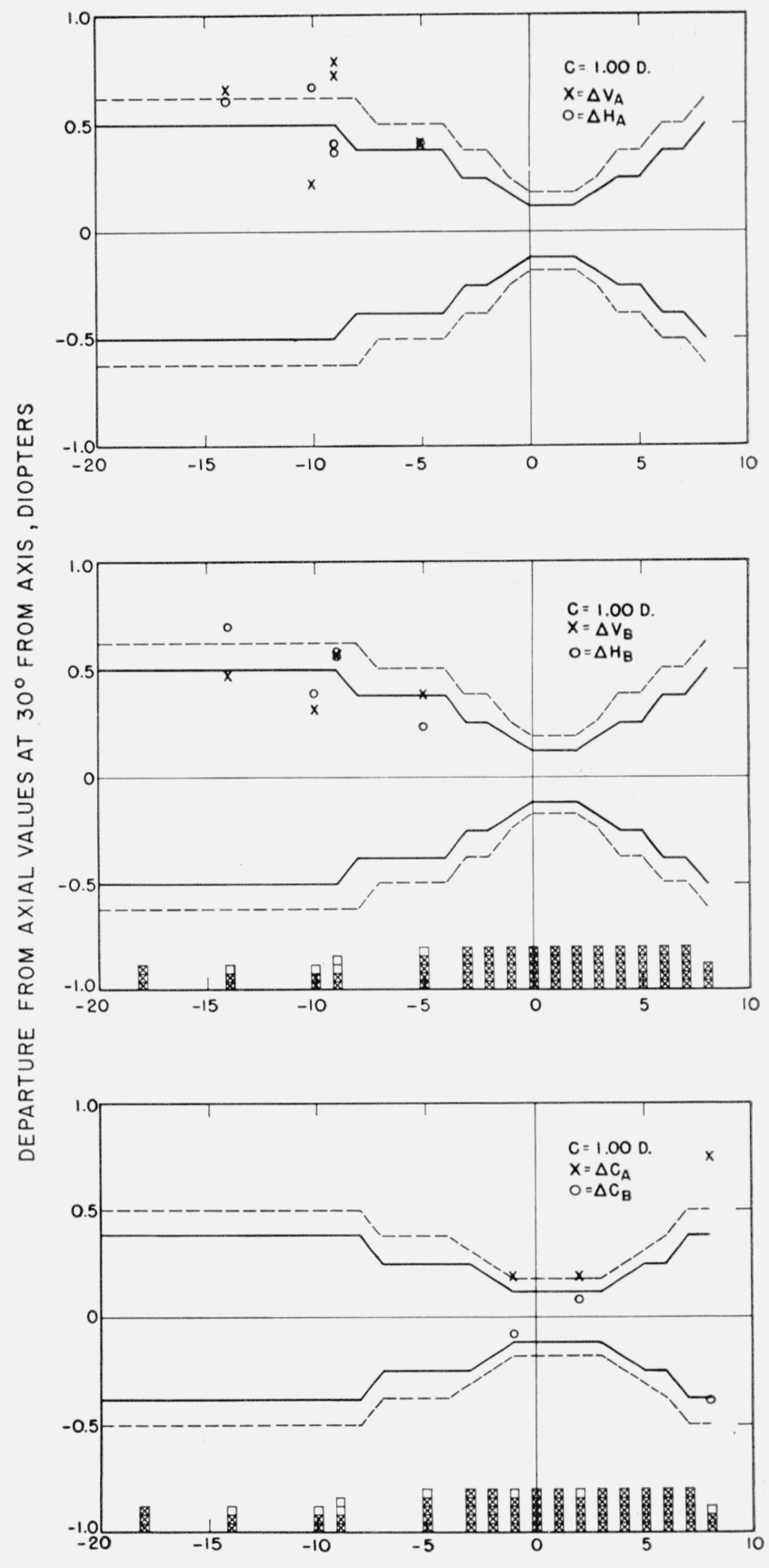

AXIAL MERIDIONAL POWER $V_{0}$, DIOPTERS

FIgURE 4. Degree of compliance with suggested tolerance for marginal power for 76 lenses having cylindrical power of 1 diopter.

Only those points are plotted for which noncompliance occurs for at least one member of a pair. The bar graphs at the bottoms of the center and lower frames member of a pair. The bar graphs at the bottoms of the center and lower frames frame she complian rame show compliance with the spherical tolerances; boxes containing an $\mathrm{X}$ in
the lower frame indicate full compliance with spherical and cylindrical tolerances

one value of the meridional power at points $A$ and $B$ is in excess of tolerance. The lowest frame shows the degree of compliance with the requirements for cylindrical power. The bar graph at the bottom of this frame combines the results for all three frames. 


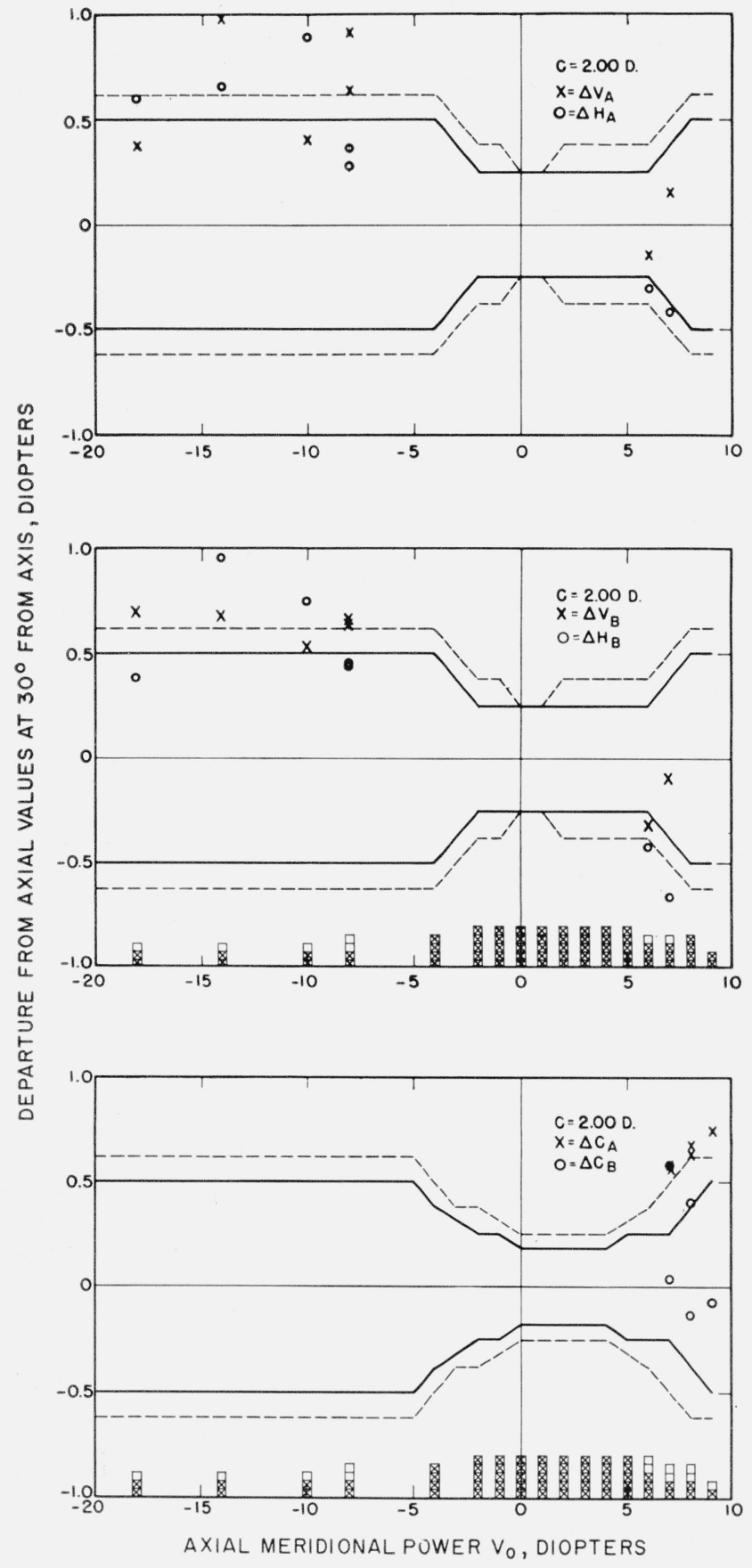

FIgure 5. Degree of compliance with suggested tolerance for marginal power for $\% 1$ lenses having cylindrical power of 2 diopters.

Only those points are plotted for which noncompliance occurs for at least one member of a pair. The bar graphs at the bottoms of the center and lower frames show the number of lenses in the sample. Boxes containing an $X$ in the center frame the lower frame indicate full compliance with spherical and cylindrical tolerances.

Consequently, a box containing an $X$ in this graph indicates that a given lens complies in full with the tolerances for meridional and cylindrical power.
It is interesting to note, that for the cases shown, at least one and generally two or more lenses comply in full with the suggested tolerances which indicate that these tolerances can be and are being satisfied. The degree of compliance with the suggested tolerances is given in tabular form in tables 16 to 20 for 295 of the 311 lenses. In these tables is given the total number of lenses of a given power in each sample, together with information on the number of lenses complying with the minimum, the maximum, and the suggested tolerances for meridional and cylindrical power in the marginal regions of the lenses. At the bottom of each table, the percentage of lenses complying in each category is given.

At first glance, the tolerances set forth in tables 11 to 15 seem larger than need be. However, the study of the degree of compliance shown in tables 16 to 20 shows that this is not the case for presently existing lenses. For example, if these lenses were to comply fully with the minimum tolerance at both points $A$ and $B$, it is clear that only a relatively small percentage of the lenses would satisfy the requirements; the percentages range from 83 percent for lenses having zero cylindrical power down to 15 percent for lenses having cylindrical power of 4.00 diopters. On the other hand, if these lenses were to comply fully with the maximum tolerance only at both points $A$ and $B$, the percentages complying range from 96 to 85 percent. However, by using the minimum tolerance at either point $A$ or point $B$ and the maximum tolerance at both points $A$ and $B$ as suggested in paragraphs (a) and (b) of section 6.1, the total number complying is reduced only slightly, the percentages ranging from 92 to 84 percent, and somewhat better performance for the user is thus assured.

TABLE 16. Degree of compliance with the minimum, maximum, and suggested tolerances for marginal power for 72 lenses having axial meridional powers varying from +7.00 to -18.00 diopters and zero cylindrical power.

\begin{tabular}{|c|c|c|c|c|}
\hline \multirow{2}{*}{$\begin{array}{l}\text { Meridional } \\
\text { power } \\
\left(V_{0}=H_{0}\right)\end{array}$} & \multirow{2}{*}{$\begin{array}{l}\text { Number of } \\
\text { lenses in } \\
\text { sample }\end{array}$} & \multicolumn{3}{|c|}{$\begin{array}{l}\text { Number of lenses in sample com- } \\
\text { plying with the following toler- } \\
\text { ances- }\end{array}$} \\
\hline & & Minimum & Maximum & Suggested \\
\hline $\begin{array}{l}7.00 \ldots \\
6.00-\ldots \\
5.00 \\
4.00 \\
3.00 \\
\end{array}$ & $\begin{array}{l}3 \\
5 \\
5 \\
5 \\
5\end{array}$ & $\begin{array}{l}2 \\
5 \\
4 \\
4 \\
4\end{array}$ & $\begin{array}{l}2 \\
5 \\
5 \\
5 \\
5\end{array}$ & $\begin{array}{l}2 \\
5 \\
5 \\
4 \\
4\end{array}$ \\
\hline $\begin{array}{r}2.00 \\
1.00 \\
0.00 \\
-1.00 \\
-2.00\end{array}$ & $\begin{array}{l}5 \\
5 \\
5 \\
5 \\
5\end{array}$ & $\begin{array}{l}5 \\
5 \\
5 \\
5 \\
5 \\
4\end{array}$ & $\begin{array}{l}5 \\
5 \\
5 \\
5 \\
5\end{array}$ & $\begin{array}{l}5 \\
5 \\
5 \\
5 \\
5 \\
5\end{array}$ \\
\hline $\begin{array}{l}-3.00 \\
-4.00 \\
-600 \\
-10.00 \\
-14.00\end{array}$ & $\begin{array}{l}5 \\
5 \\
5 \\
5 \\
3 \\
3\end{array}$ & $\begin{array}{l}4 \\
3 \\
3 \\
2 \\
2 \\
2\end{array}$ & $\begin{array}{l}5 \\
5 \\
4 \\
3 \\
2\end{array}$ & $\begin{array}{l}5 \\
4 \\
4 \\
3 \\
2 \\
2\end{array}$ \\
\hline$-18.00 \ldots$ & 3 & 3 & 3 & 3 \\
\hline $\begin{array}{l}\text { Total_-... } \\
\text { Percentage }\end{array}$ & 72 & $\begin{array}{l}60 \\
83\end{array}$ & $\begin{array}{l}69 \\
96\end{array}$ & $\begin{array}{l}66 \\
92\end{array}$ \\
\hline
\end{tabular}


TABLE 17. Degree of compliance with the minimum, maximum, and suggested tolerances for marginal power for 76 lenses having axial meridional powers varying from +8.00 to -18.00 diopters and cylindrical power of +1.00 diopter

\begin{tabular}{|c|c|c|c|c|}
\hline \multirow{2}{*}{$\begin{array}{c}\text { Meridional } \\
\text { power } \\
\left(V_{O}=\right. \\
\left.H_{O}+1.00\right)\end{array}$} & \multirow{2}{*}{$\begin{array}{l}\text { Number of } \\
\text { lenses in } \\
\text { sample }\end{array}$} & \multicolumn{3}{|c|}{$\begin{array}{l}\text { Number of lenses in sample comply- } \\
\text { ing with the following tolerances- }\end{array}$} \\
\hline & & Minimum & Maximum & Suggested \\
\hline $\begin{array}{l}8.00 \\
7.00 \\
6.00 \\
5.00 \\
4.00\end{array}$ & $\begin{array}{l}3 \\
5 \\
5 \\
5 \\
5\end{array}$ & $\begin{array}{l}1 \\
4 \\
3 \\
4 \\
3\end{array}$ & $\begin{array}{l}2 \\
5 \\
5 \\
5 \\
5\end{array}$ & $\begin{array}{l}2 \\
5 \\
5 \\
5 \\
5\end{array}$ \\
\hline $\begin{array}{r}3.00 \\
2.00 \\
1.00 \\
0.00 \\
-1.00\end{array}$ & $\begin{array}{l}5 \\
5 \\
5 \\
5 \\
5\end{array}$ & $\begin{array}{l}5 \\
2 \\
2 \\
5 \\
5 \\
2\end{array}$ & $\begin{array}{l}5 \\
4 \\
5 \\
5 \\
4\end{array}$ & $\begin{array}{l}5 \\
4 \\
5 \\
5 \\
4\end{array}$ \\
\hline $\begin{array}{l}-2.00 \\
-3.00 \\
-5.00 \\
-9.00 \\
-10.00\end{array}$ & $\begin{array}{l}5 \\
5 \\
5 \\
4 \\
3\end{array}$ & $\begin{array}{l}5 \\
3 \\
3 \\
2 \\
2 \\
2\end{array}$ & $\begin{array}{l}5 \\
5 \\
5 \\
2 \\
2 \\
2\end{array}$ & $\begin{array}{l}5 \\
5 \\
4 \\
2 \\
2\end{array}$ \\
\hline $\begin{array}{l}-14.00 \\
-18.00\end{array}$ & $\begin{array}{l}3 \\
3\end{array}$ & $\begin{array}{l}1 \\
3\end{array}$ & $\stackrel{2}{3}$ & $\begin{array}{l}2 \\
3\end{array}$ \\
\hline $\begin{array}{l}\text { Total_} \\
\text { Percentage }\end{array}$ & 76 & $\begin{array}{l}53 \\
70\end{array}$ & $\begin{array}{l}69 \\
91\end{array}$ & $\begin{array}{l}68 \\
90\end{array}$ \\
\hline
\end{tabular}

TABLE 18. Degree of compliance with the minimum, maximum, and suggested tolerances for marginal power for 71 lenses having axial meridional powers varying from +9.00 to -18.00 diopters and cylindrical power of +2.00 diopters

\begin{tabular}{|c|c|c|c|c|}
\hline \multirow{2}{*}{$\begin{array}{c}\text { Meridional } \\
\text { power } \\
\text { (Vo= } \\
\left.H_{O}+2.00\right)\end{array}$} & \multirow{2}{*}{$\begin{array}{c}\text { Number of } \\
\text { lenses in } \\
\text { sample }\end{array}$} & \multicolumn{3}{|c|}{$\begin{array}{l}\text { Number of lenses in sample comply- } \\
\text { ing with the following tolerances- }\end{array}$} \\
\hline & & Minimum & Maximum & Suggested \\
\hline $\begin{array}{l}9.00 \\
8.00 \\
7.00 \\
6.00 \\
5.00\end{array}$ & $\begin{array}{l}2 \\
4 \\
4 \\
4 \\
5\end{array}$ & $\begin{array}{l}0 \\
0 \\
0 \\
0 \\
0\end{array}$ & $\begin{array}{l}1 \\
2 \\
2 \\
3 \\
5\end{array}$ & $\begin{array}{l}1 \\
2 \\
2 \\
3 \\
5 \\
5\end{array}$ \\
\hline $\begin{array}{l}4.00 \\
3.00 \\
2.00 \\
1.00 \\
0.00\end{array}$ & $\begin{array}{l}5 \\
5 \\
5 \\
5 \\
5\end{array}$ & $\begin{array}{l}2 \\
4 \\
5 \\
2 \\
1\end{array}$ & $\begin{array}{l}5 \\
5 \\
5 \\
5 \\
5\end{array}$ & $\begin{array}{l}5 \\
5 \\
5 \\
5 \\
5\end{array}$ \\
\hline $\begin{array}{l}-1.00 \\
-2.00 \\
-4.00 \\
-8.00 \\
-10.00\end{array}$ & $\begin{array}{l}5 \\
5 \\
4 \\
4 \\
3\end{array}$ & $\begin{array}{l}1 \\
0 \\
3 \\
2 \\
2\end{array}$ & $\begin{array}{l}5 \\
5 \\
4 \\
2 \\
2 \\
2\end{array}$ & $\begin{array}{l}5 \\
5 \\
4 \\
2 \\
2 \\
2\end{array}$ \\
\hline $\begin{array}{l}-14.00 \\
-18.00\end{array}$ & $\begin{array}{l}3 \\
3\end{array}$ & $\begin{array}{l}1 \\
1\end{array}$ & $\stackrel{2}{2}$ & $\begin{array}{l}2 \\
2\end{array}$ \\
\hline $\begin{array}{l}\text { Total } \\
\text { Percentage- }\end{array}$ & 71 & $\begin{array}{l}24 \\
34\end{array}$ & $\begin{array}{l}60 \\
85\end{array}$ & $\begin{array}{l}60 \\
85\end{array}$ \\
\hline
\end{tabular}

\subsection{Discussion of the Proposed Tolerances}

In the preparation of tolerances for axial powers, it is possible to require that the deviation of measured from prescribed power be kept within a narrow enough range that normal depth of focus will still enable the unaccommodated eye to distinguish objects separated by 1 minute of arc. However, in the case of tolerances for marginal power, it is not readily possible to maintain such a high degree of performance because of limitations imposed by having only two surfaces available to the designer, conditions of use, and the cost. Although it is possible to design a lens for each power that will perform in excellent fashion for one viewing dis-
TABLE 19. Degree of compliance with the minimum, maximum, and suggested tolerances for marginal power for 43 lenses having axial meridional powers varying from +9.00 to -7.00 diopters and cylindrical power of +3.00 diopters

\begin{tabular}{|c|c|c|c|c|}
\hline \multirow{2}{*}{$\begin{array}{c}\text { Meridional } \\
\text { power } \\
\left(V_{0}=\right. \\
\left.H_{O}+3.00\right)\end{array}$} & \multirow{2}{*}{$\begin{array}{l}\text { Number of } \\
\text { lenses in } \\
\text { sample }\end{array}$} & \multicolumn{3}{|c|}{$\begin{array}{l}\text { Number of lenses in sample comply- } \\
\text { ing with the following tolerances- }\end{array}$} \\
\hline & & Minimum & Maximum & Suggested \\
\hline $\begin{array}{l}9.00 \\
8.00 \\
7.00 \\
6.00 \\
2.00\end{array}$ & $\begin{array}{l}4 \\
4 \\
4 \\
4 \\
5\end{array}$ & $\begin{array}{l}0 \\
0 \\
0 \\
2 \\
3\end{array}$ & $\begin{array}{l}3 \\
3 \\
3 \\
4 \\
4 \\
5\end{array}$ & $\begin{array}{l}3 \\
2 \\
2 \\
4 \\
5 \\
5\end{array}$ \\
\hline $\begin{array}{r}1.00 \\
0.00 \\
-1.00 \\
-3.00 \\
-7.00\end{array}$ & $\begin{array}{l}5 \\
5 \\
4 \\
4 \\
4 \\
4\end{array}$ & $\begin{array}{l}2 \\
1 \\
0 \\
1 \\
1\end{array}$ & $\begin{array}{l}5 \\
5 \\
4 \\
4 \\
4 \\
\end{array}$ & $\begin{array}{l}5 \\
5 \\
4 \\
4 \\
2 \\
2\end{array}$ \\
\hline $\begin{array}{l}\text { Total } \\
\text { Percentage. }\end{array}$ & 43 & $\begin{array}{l}10 \\
23\end{array}$ & $\begin{array}{l}40 \\
93\end{array}$ & $\begin{array}{l}36 \\
84\end{array}$ \\
\hline
\end{tabular}

TABLE 20. Degree of compliance with the minimum, maximum, and suggested tolerances for marginal power for 33 lenses having axial meridional powers varying from +9.00 to -6.00 diopters and cylindrical power of +4.00 diopters

\begin{tabular}{|c|c|c|c|c|}
\hline \multirow{2}{*}{$\begin{array}{c}\text { Meridional } \\
\text { power } \\
\left(V_{o}=\right. \\
\left.H_{O}+4.00\right)\end{array}$} & \multirow{2}{*}{$\begin{array}{l}\text { Number of } \\
\text { lenses in } \\
\text { sample }\end{array}$} & \multicolumn{3}{|c|}{$\begin{array}{l}\text { Number of lenses in sample comply- } \\
\text { ing with the following tolerances- }\end{array}$} \\
\hline & & Minimum & Maximum & Suggested \\
\hline $\begin{array}{l}9.00 \\
8.00 \\
3.00 \\
2.00\end{array}$ & $\begin{array}{l}4 \\
4 \\
4 \\
4\end{array}$ & $\begin{array}{l}0 \\
0 \\
1 \\
1\end{array}$ & $\begin{array}{l}2 \\
3 \\
4 \\
4\end{array}$ & $\begin{array}{l}2 \\
2 \\
4 \\
4\end{array}$ \\
\hline $\begin{array}{r}1.00 \\
0.00 \\
-2.00 \\
-6.00\end{array}$ & $\begin{array}{l}4 \\
5 \\
4 \\
4\end{array}$ & $\begin{array}{l}0 \\
0 \\
1 \\
2\end{array}$ & $\begin{array}{l}4 \\
5 \\
4 \\
4 \\
4\end{array}$ & $\begin{array}{l}3 \\
5 \\
4 \\
4\end{array}$ \\
\hline $\begin{array}{l}\text { Total_...... } \\
\text { Percentage. }\end{array}$ & 33 & $\begin{array}{r}5 \\
15\end{array}$ & $\begin{array}{l}30 \\
91\end{array}$ & $\begin{array}{l}28 \\
85\end{array}$ \\
\hline
\end{tabular}

tance, it is not likely that it will perform equally well for all other viewing distances. In addition, it is impracticable because of cost to construct a special lens for each user, so it is usual to maintain a series of lenses whose base-curve changes in successive discrete steps to cover the entire range of use.

The suggested tolerances for spherical power do not present any serious hardship for the wearer under middle age, as such a person is likely to have an amplitude of accommodation well above the maximum suggested tolerances. For a person having a small amplitude of accommodation, the marginal performance of a lens near the limits of the suggested tolerance will probably be noticeably lower than the axial performance. However, he will still usually be able to distinguish between objects separated by from 2 to 8 minutes of arc, depending upon the amount of departure of the marginal from the axial powers. This corresponds to a reduction in visual acuity down to $20 / 40$ or $20 / 160$, which is still adequate for the detection of large objects or movement.

In the case of astigmatism, the problem is more difficult. One cannot minimize astigmatism to an appreciable extent by the exercise of accommodative powers. Consequently, all users will suffer some reduction in visual acuity in the marginal regions, 


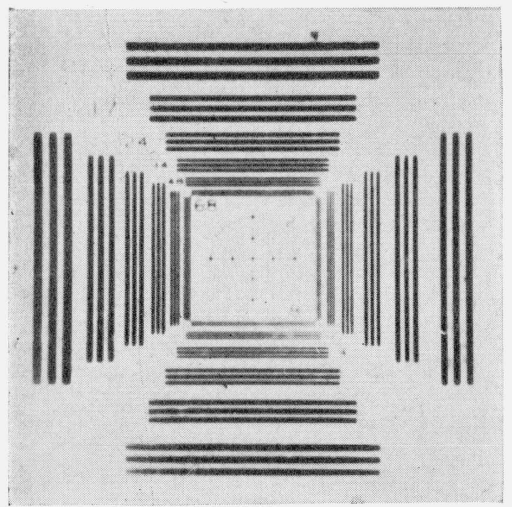

A

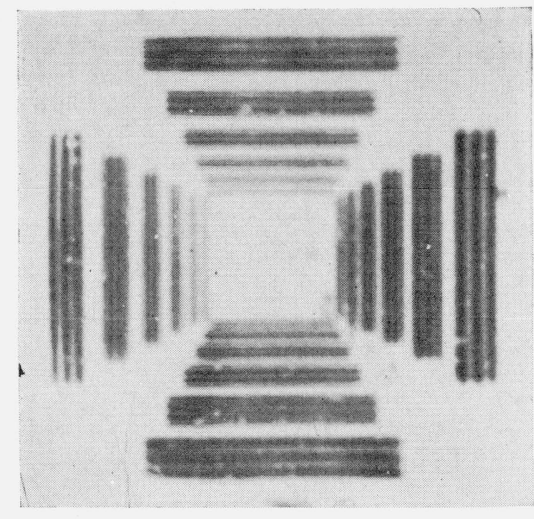

B

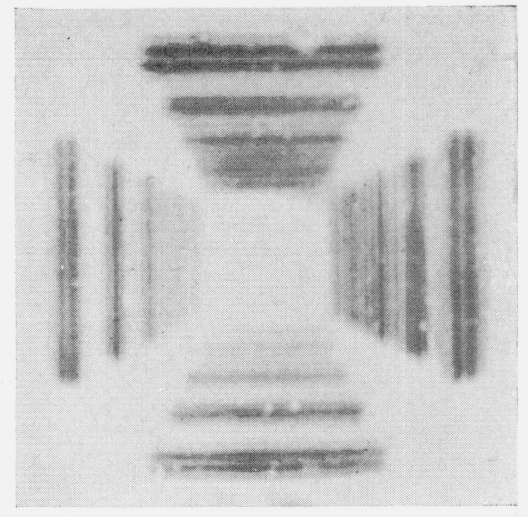

C

Figure 6. Photographs showing change in imagery with departures from prescribed spherical power by definite amounts.

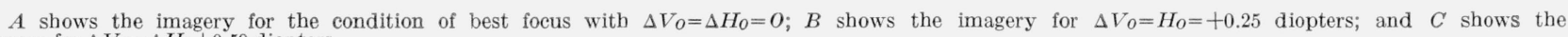
imagery for $\Delta V_{O}=\Delta H_{O}+0.50$ diopters.

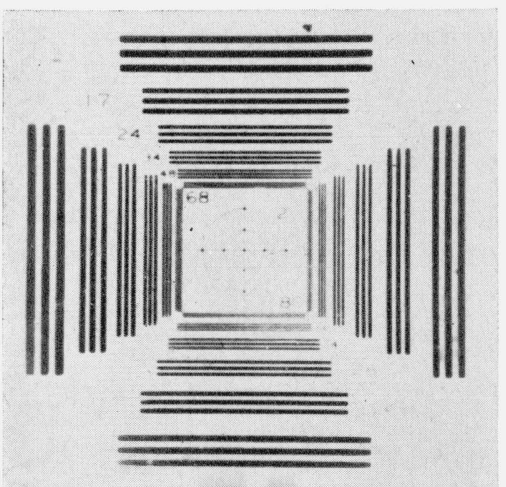

A

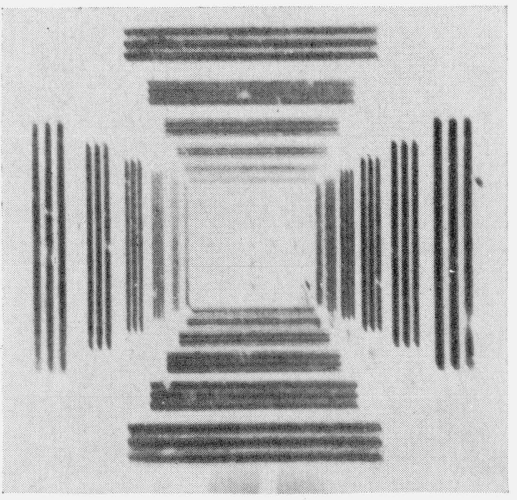

B

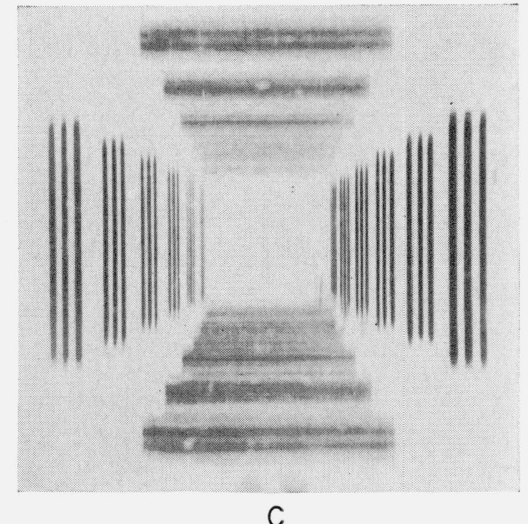

C

Figure 7. Photographs showing change in imagery with departures from prescribed cylindrical power by definite amounts.

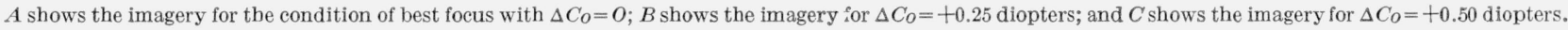

except in those instances where the depth of focus is sufficient to provide adequate compensation. Of course, it is unlikely that anyone will be using the marginal portion of his spectacle lenses for an extended time, as it is more natural to turn the head so as to take advantage of central vision. Figures 6 and 7 show the quality of imagery that may be expected when looking through lenses whose powers vary from the prescribed by selected amounts [5].

In conclusion, it can be stated that there are now available a large number of lenses that are corrected to yield improved marginal performance, and that they do so. However, further efforts toward improvement should be made. If this is done, it may eventually be possible to reduce the tolerances suggested herein to values more nearly comparable to the depth of focus.

\section{References}

[1] F. E. Washer, Axial performance of spectacle lenses, J. Research NBS 57, 355 (1956) RP2724.

[2] F. E. Washer, A simplified method of measuring the marginal power of spectacle lenses, J. Research NBS 55, 79 (1955) RP2607.

[3] H. H. Emsley and William Swaine, Ophthalmic lenses, 6th ed., p. 237 (Hatton Press Ltd., London, 1951).

[4] Veterans Administration, specifications for optical lenses, frames and cases (April 23, 1956).

[5] F. E. Washer, Instrument for measuring the marginal power of spectacle lenses, J. Opt. Soc. Am. 45, 719 (1955).

Washington, November 21, 1957 